KYUSHU-HET-71

hep-ph/0311337

\title{
Sparticle Masses in Product-Group Gauge Theories
}

\author{
Nobuhito Maru* and Koichi Yoshioka ${ }^{\dagger}$ \\ * Theoretical Physics Laboratory, \\ RIKEN (The Institute of Physical and Chemical Research), \\ 2-1 Hirosawa, Wako, Saitama 351-0198, Japan \\ †Department of Physics, Kyushu University, Fukuoka 812-8581, Japan
}

\begin{abstract}
In this paper, we investigate a possibility to cause supersymmetry breaking with background modulus fields in product-group gauge theories. The vacuum expectation values of these moduli are found to satisfy several relations and the moduli dependences of the action are fixed by consistency of the model. With this action, we calculate the mass spectrum of vector and matter multiplets up to one-loop order of perturbation theory. As an application of the result, it is found that various properties of higher-dimensional supersymmetry breaking are well captured in corresponding limits in the moduli space. In particular, we have finite radiative corrections to Higgs masses in the case that is closely related to the boundary condition breaking of supersymmetry.
\end{abstract}

*E-mail address: maru@postman.riken.go.jp, Special Postdoctoral Researcher

${ }^{\dagger}$ E-mail address: yoshioka@higgs.phys.kyushu-u.ac.jp 


\section{Introduction}

Motivated by the gauge hierarchy problem, physics with extra dimensions has provided new insights on various aspects of particle physics, cosmology and astrophysics [1,2]. There however seems to be some difficulty to discuss the issues in the ultraviolet regime since higherdimensional theories are generally non-renormalizable in the usual sense. Recently, a fourdimensional framework describing physics of higher-dimensional theories has been proposed in $[3,4]$. The framework consists of a gauge theory of product group and scalar fields in the bi-fundamental representations of the nearest-neighbor gauge groups. If one assumes that the bi-fundamental scalars develop vacuum expectation values (VEVs), the gauge groups are broken to a simple diagonal subgroup. It has been shown that with a sufficiently large number of gauge groups, the mass spectrum of gauge fields is equivalent in an intermediate energy regime to Kaluza-Klein (KK) mass spectrum of a five-dimensional gauge theory. This approach is providing new tools for four-dimensional model building and moreover for understanding unexplored properties of higher-dimensional theories. In fact, various applications along this line has been discussed in the literature [5].

In Ref. [6], we have studied a model with supersymmetry (SUSY) breaking induced by modulus fields which are naturally incorporated into the above framework. The modulus fields are found to satisfy some relations and have non-trivial dependences of the action, if one wants to obtain a description of higher-dimensional effects. We have identified the modulus form of the action and verified it for a five-dimensional vector multiplet by explicitly calculating tree-level mass spectrum of gaugino and associated adjoint scalar. Various types of SUSYbreaking mass spectra predicted in higher-dimensional models appear as corresponding limits in the parameter space of the modulus $F$ terms.

In this paper, as an extension of the previous results, we formulate a four-dimensional model with general matter multiplets and evaluate one-loop radiative corrections to mass spectrum. We first fix the modulus couplings to matter multiplets as well as to vector ones, and then calculate radiative corrections to gaugino and scalar soft masses in detail. It will be found that the above-mentioned resemblances to the existing higher-dimensional models still hold even at quantum level. In addition, we study the ultraviolet behavior of the radiative corrections and find in some case the corrections to Higgs masses to be finite. Such a case is explicitly shown to be closely related to the boundary condition breaking of supersymmetry.

The paper is organized as follows. In Section 2, we first review the action for vector multiplets and the resulting tree-level mass spectrum of gauginos and adjoint scalars. We also fix the modulus couplings to matter multiplets and calculate the (SUSY-breaking) masses at classical level. With the complete action at hand, radiative corrections to the masses of vector and matter multiplets are studied in detail in Section 3. Section 4 gives some arguments about the finiteness property of loop corrections found in Section 3. There we will particularly pay attention to some relation between the finite corrections and the boundary condition breaking of supersymmetry. Section 5 is devoted to the summary of the paper. 


\section{Classical action}

\section{$2.1 \quad$ Vector multiplets}

The model we consider is a four-dimensional SUSY gauge theory with the gauge groups $G_{1} \times$ $G_{2} \times \cdots \times G_{N}$. We simply assume all the gauge couplings $g_{i}$ to have the universal value $g$. The four-dimensional $N=1$ vector multiplet $V_{i}$ of the $G_{i}$ gauge theory contains a gauge field $A_{\mu}^{i}$ and a gaugino $\lambda^{i}$. In addition, we have the $N=1$ matter multiplets $Q_{i}(i=1, \cdots, N)$ in the bi-fundamental representation, that is, $Q_{i}$ transforms as $(\square, \bar{\square})$ under the $\left(G_{i}, G_{i+1}\right)$ gauge groups. $\left(G_{N+1}\right.$ is identified to $G_{1}$.) The field content of the model is then summarized below:

\begin{tabular}{c|ccccc} 
& $G_{1}$ & $G_{2}$ & $G_{3}$ & $\cdots$ & $G_{N}$ \\
\hline$Q_{1}$ & $\square$ & $\bar{\square}$ & 1 & $\cdots$ & 1 \\
$Q_{2}$ & 1 & $\square$ & $\bar{\square}$ & $\cdots$ & 1 \\
$\vdots$ & $\vdots$ & $\vdots$ & $\vdots$ & $\ddots$ & $\vdots$ \\
$Q_{N}$ & $\bar{\square}$ & 1 & 1 & $\cdots$ & $\square$
\end{tabular}

The gauge-invariant Lagrangian for vector multiplets and $Q_{i}$ 's is written as

$$
\mathcal{L}_{\text {vec }}=\sum_{i}\left[\int d^{2} \theta S W_{i}^{\alpha} W_{\alpha i}+\text { h.c. }+\int d^{2} \theta d^{2} \bar{\theta} K_{Q}\left(S, S^{\dagger}\right) Q_{i}^{\dagger} e^{\sum V} Q_{i}\right],
$$

where $W_{i}^{\alpha}$ is the field strength superfield of the $G_{i}$ gauge group, and $S$ is a dilaton-like background superfield whose scalar component determines a value of $g$ [see Eq. (2.6)]. In fact, one may introduce a dilaton field for each gauge theory $G_{i}$. We now take the universal value of gauge couplings and use $S$ as a representative of such dilaton fields. The normalization function $K_{Q}\left(S, S^{\dagger}\right)$ of the matter field $Q_{i}$ is found [6]

$$
K_{Q}\left(S, S^{\dagger}\right)=\frac{8}{1 / S+1 / S^{\dagger}}
$$

which can be fixed by several classical-level consistencies as will be discussed at the end of this section. Moreover, this form of $K_{Q}$ will be found in the next section to be also important for loop-level consistency of the model.

If one considers the case that $Q_{i}$ 's have VEVs proportional to the unit matrix, $\left\langle Q_{i}\right\rangle=v$, ${ }^{*}$ the gauge symmetries are broken down to its diagonal subgroup $G$. With this symmetry breaking, the mass eigenvalues and eigenstates of the gauge fields are given by

$$
\begin{aligned}
& m_{n}=2 v \sin \frac{n \pi}{N}, \\
& \widetilde{A}_{\mu}^{n}=\frac{1}{\sqrt{N}} \sum_{j=1}^{N}\left(\omega_{n}\right)^{j} A_{\mu}^{j}, \quad(n=0, \cdots, N-1)
\end{aligned}
$$

${ }^{*}$ More generic cases with non-universal values of the gauge couplings and VEVs of $Q$ 's correspond to gauge theories on curved backgrounds [7] and low-energy gauge symmetry breaking [8]. We do not consider these possibilities in the present work. 
where $\omega_{n}=e^{2 \pi i n / N}$. We thus find the KK tower of massive gauge fields as well as a massless one which belongs to the unbroken subgroup $G$. It is interesting to note that the spectrum matches to that of a five-dimensional gauge theory with a compact extra dimensions [3, 4]. That is, in the large $N$ limit with $v / N$ fixed, the gauge boson masses approximately become

$$
m_{n} \simeq \frac{2 v n \pi}{N}=\frac{n}{R}
$$

where the compactification radius $R$ is identified as $2 \pi R=N / v$. With this identification, $v$ is interpreted as an ultraviolet cutoff of the effective four-dimensional theory, and $N$ is the number of KK modes which exist between the cutoff and compactification scales.

In the absence of SUSY breaking, the gauginos and the associated adjoint scalars, which are linear combinations of the original fields $\lambda_{i}$ and $Q_{i}$, have degenerate mass spectrum with that of the gauge bosons (2.4). Before examining SUSY-breaking mass splitting, it may be instructive to recall the modulus fields in our model and their mutual relations. In addition to $S$ introduced above, there is a modulus $Q$ which determines the size of the universal VEV $v$. The modulus $Q$ may be a normalized composite field or a linear combination of $Q_{i}$ 's. Throughout this paper, we assume just for simplicity that the moduli forms of $S_{i}$ and $Q_{i}$ are invariant under the "translation" transverse to the four dimensions, that is, they are $i$-independent. The forms of VEVs of these moduli are then defined as

$$
S=\frac{1}{4 g^{2}}+F_{S} \theta^{2}, \quad Q=v+F_{Q} \theta^{2}
$$

Furthermore, it will turn out to be useful to define the additional background modulus fields with the following VEVs

$$
S_{4}=\frac{1}{4 g_{4}^{2}}+F_{S 4} \theta^{2}, \quad S_{5}=\frac{1}{4 g_{5}^{2}}+F_{S 5} \theta^{2}, \quad T=\frac{1}{R}+F_{T} \theta^{2},
$$

where $g_{4}$ and $g_{5}$ are the effective gauge coupling constants in four and five dimensions, respectively. It is important to notice that all these modulus fields are not independent variables. By comparing the Kaluza-Klein theory with the low-energy description of the model below the cutoff $v$, one finds the following matching conditions between the parameters $[3,4]$ :

$$
2 \pi R=\frac{N}{v}, \quad g_{4}^{2}=\frac{g^{2}}{N} .
$$

The first condition in (2.8) was already adopted in (2.5), which was required to match the spectrum to that of KK theory. The second condition is regarded as a volume suppression of bulk gauge coupling in compactifying an extra dimension. In addition to these, the normalization of gauge kinetic terms provides a relation between $g_{4}$ and $g_{5}$, irrespectively of how to define a five-dimensional model,

$$
g_{5}^{2}=2 \pi R g_{4}^{2}
$$


These three relations among the couplings suggest the following relations among the modulus fields: ${ }^{\dagger}$

$$
S_{4}=N S, \quad S_{5}=Q S, \quad T=\frac{2 \pi}{N} Q
$$

We thus find that the modulus fields $S_{4}, S_{5}$ and $T$ are expressed in terms of two moduli $S$ and $Q$. As a result, the two-dimensional parameter space of the $F$-components of $S$ and $Q$ describes SUSY-breaking patterns in our model. In Ref. [6], we clarified that several limits in this parameter space describe bulk SUSY-breaking patterns which have been discussed in the literature ${ }^{\ddagger}$ In Table 1, the typical cases are presented for the dilaton/moduli dominated SUSY-breaking [10] and for SUSY breaking by the radius modulus $F$ term [11, 12]. The specification of each limit is also given in the table. For example, the dilaton dominance scenario is defined by $F_{T}=0$, which is in turn translated to the limit $F_{Q}=0$ in our model [see Eq. (2.10)]. In the following, we will study the SUSY-breaking effects from these modulus

\begin{tabular}{lccccc}
\hline \hline & $F_{S}$ & $F_{Q}$ & $F_{S 4}$ & $F_{S 5}$ & $F_{T}$ \\
\hline $\begin{array}{l}\text { dilaton } \\
\left(F_{T} \equiv 0\right)\end{array}$ & $F_{S}$ & 0 & $N F_{S}$ & $v F_{S}$ & 0 \\
moduli & 0 & $F_{Q}$ & 0 & $\frac{1}{4 g^{2}} F_{Q}$ & $\frac{2 \pi}{N} F_{Q}$ \\
$\left(F_{S 4} \equiv 0\right)$ & $-F_{Q}$ & $F_{Q}$ & $\frac{-N F_{Q}}{4 g^{2} v}$ & 0 & $\frac{2 \pi}{N} F_{Q}$ \\
$\begin{array}{l}\text { radion } \\
\left(F_{S 5} \equiv 0\right)\end{array}$ & $\frac{4 g^{2} v}{10}$ & & & \\
\hline \hline
\end{tabular}

Table 1: The modulus F-terms of the typical SUSY-breaking scenarios.

fields and examine sparticle spectrum of the model.

If one introduces suitable potential terms for the modulus fields, their VEVs are fixed to some point in the parameter space. For example, since $S$ is a dilaton for each gauge group, stabilization mechanisms proposed in the literature could be incorporated in the present model. The situation is similar for the modulus $Q$. Moreover in properly describing five-dimensional theory, $Q$ is assumed to be stabilized by relevant potential terms [3, 9]. However details of potential form are not relevant to the present analysis. Without referring to specific models, we explore the whole parameter space of the modulus $F$-terms and then examine several limits corresponding to bulk SUSY-breaking patterns. Moreover we do not try to construct specific dynamics for the modulus fields where potential couplings are tuned for realizing the fifth dimension. Our aim here is not to present five-dimensional theories. It is only the relevant region of moduli space where our model reproduces bulk SUSY-breaking scenarios. In other words, the present framework contains unexplored four-dimensional phenomena of SUSY breaking.

\footnotetext{
$\dagger$ The 1PI and holomorphic gauge couplings differ only at higher-loop level in perturbation theory.

${ }^{\ddagger}$ Supersymmetry breaking that is local in the higher-dimensional bulk was studied within four-dimensional product-group gauge theories [9].
} 
Now the SUSY-breaking mass spectrum can be derived from the Lagrangian (2.2) with turning on the modulus $F$ terms. The result is written by use of the above-defined moduli and their relations;

$$
\begin{aligned}
m_{\lambda_{n \pm}} & = \pm \sqrt{m_{n}^{2}+\left|\frac{F_{S 5}}{2\left\langle S_{5}\right\rangle}\right|^{2}}-\frac{F_{T}}{2\langle T\rangle} \\
m_{c_{n}}^{2} & =m_{n}^{2}+2 \operatorname{Re}\left(\frac{F_{S 5}^{*}}{\left\langle S_{5}\right\rangle} \frac{F_{T}}{\langle T\rangle}\right)
\end{aligned}
$$

where $m_{\lambda_{n \pm}}$ and $m_{c_{n}}$ are the KK masses of gauge fermions and adjoint scalars. The bracket \langle\rangle denotes a VEV of the lowest scalar component. The positive (negative) sign in $m_{\lambda_{n \pm}}$ corresponds to the gaugino (the goldstone fermion) masses in the supersymmetric limit. Note that the results are expressed by five-dimensional quantities only. In particular, it is found the zero-mode gaugino mass is given by both the radius modulus and the five-dimensional dilaton. This result is expected from the equation (2.9) which implies $S_{4}$ depends both on $T$ and $S_{5}$ $\left(T S_{4}=2 \pi S_{5}\right)$.

We here explicitly show the several limits in order. As mentioned before, the dilatondominated SUSY breaking is characterized by the condition $F_{T}=0$. In this limit, we find

$$
m_{\lambda_{n \pm}}= \pm \sqrt{m_{n}^{2}+\left|2 g^{2} F_{S}\right|^{2}}, \quad m_{c_{n}}^{2}=m_{n}^{2} . \quad \text { [dilaton] }
$$

The gaugino mass spectrum is indeed the one expected in supergravity models. All the KK states including the zero mode receive the universal SUSY-breaking contribution from the modulus $S$, the two level- $n$ spinors are degenerate in mass, and the mass splitting between bosons and fermions are equal for all KK modes. These results may be understood from the fact that the dilaton field commonly couples to any field in the theory.

On the other hand, the limit of the moduli-dominated SUSY breaking is defined by $F_{S_{4}}=0$ and then leads to

$$
m_{\lambda_{n \pm}}= \pm \sqrt{m_{n}^{2}+\left|\frac{F_{Q}}{2 v}\right|^{2}}-\frac{F_{Q}}{2 v}, \quad m_{c_{n}}^{2}=m_{n}^{2}+2\left|\frac{F_{Q}}{v}\right|^{2} . \quad \text { [moduli] }
$$

It is interesting to note that even when the SUSY-breaking effect is turned on, the zero-mode gaugino remains massless. ${ }^{\S}$ This is exactly the tree-level spectrum predicted in this class of SUSY-breaking models $[10,13]$. Since $F_{S 4}=0$ by definition, the zero-mode gaugino mass is vanishing and is shifted at loop level by string threshold corrections or effects of bulk fields. The situation is also similar to the model where vector multiplets behave as messengers of SUSY breaking and sparticle soft masses are calculated from the wave function renormalization in four dimensions [14]. There may be an intuitive explanation for the above type of spectrum. That is, a nonzero $F$ term of the modulus which determines KK masses does not induce tree-level SUSY-breaking masses for zero modes. This is because these two mass terms are

\footnotetext{
$\S$ The level $n=0$ spinor being affected by the non-zero $F$-terms is the goldstone fermion associated to gauge symmetry breaking.
} 
proportional to KK numbers. In our case, such a modulus corresponds to the one whose scalar component obtains a $\mathrm{VEV} \propto 1 / R$ and is given by $Q \propto T$.

The last example $F_{S_{5}}=0$ realizes the SUSY breaking with the radius modulus field. The sparticle mass spectrum in this limit becomes

$$
m_{\lambda_{n \pm}}= \pm m_{n}-\frac{F_{T}}{2\langle T\rangle}, \quad m_{c_{n}}^{2}=m_{n}^{2} . \quad \text { [radion] }
$$

The gaugino mass matches to that calculated in [12]. The vanishing SUSY-breaking masses of the adjoint scalars agree with the fact that this limit is equivalent to the Scherk-Schwarz mechanism [15], which is now applied to the $S U(2)_{R}$ symmetry under which the adjoint scalar is singlet and does not get a symmetry breaking mass.

Before closing this subsection, we comment on the normalization function $K_{Q}\left(S, S^{\dagger}\right)$. The form of $K_{Q}(2.3)$ is determined so that it satisfies several nontrivial requirements. First, the holomorphy requires the normalization of $Q_{i}$ to be $\left\langle K_{Q}\right\rangle=1 / g^{2}$, which leads to the same normalization for the vector and adjoint chiral multiplets of the low-energy $G$ gauge theory. Moreover, the radius superfield in our model becomes independent of the dilaton superfield [see (2.10)]. It seems plausible since an undesirable relation between the theta angle and the graviphoton field does not arise. Another consistency is about the 5-5 component of fivedimensional metric $g_{55}$. In a continuum five-dimensional theory, the kinetic terms of bosonic fields along the fifth dimension have a dependence of $g_{55}$ as $\sqrt{g_{55}} g^{55} \propto 1 / R$. In the present model, the second term in the Lagrangian (2.2) becomes the kinetic energy in the continuum limit and its modulus dependence is given by $\left\langle K_{Q}\left(S, S^{\dagger}\right) Q^{\dagger} Q\right\rangle$. The equation (2.3) then indicates $\left\langle K_{Q} Q^{2}\right\rangle \sim\left\langle S Q^{2}\right\rangle \sim\left\langle S_{5} T\right\rangle$. Consequently, the metric dependence agrees for a fixed value of the five-dimensional coupling $g_{5}$.

\subsection{Matter multiplets}

Next let us discuss matter multiplets, which are regarded as hypermultiplets if one takes the five-dimensional limit. As in the case of vector multiplets, we will determine the proper form of moduli dependences of the matter action and then examine SUSY-breaking mass spectrum at tree level. One-loop corrections to the masses of scalar components in these matter multiplets will be studied in Section 3 .

In addition to the fields in the previous subsection, we introduce a set of vector-like chiral multiplets $\Phi_{i}$ and $\bar{\Phi}_{i}$ for each gauge group $G_{i}$ :

\begin{tabular}{c|ccccc} 
& $G_{1}$ & $G_{2}$ & $G_{3}$ & $\ldots$ & $G_{N}$ \\
\hline$\Phi_{1}$ & $\square$ & 1 & $\ldots$ & $\ldots$ & 1 \\
$\bar{\Phi}_{1}$ & $\bar{\square}$ & 1 & $\ldots$ & $\ldots$ & 1 \\
$\Phi_{2}$ & 1 & $\square$ & $\ldots$ & $\ldots$ & 1 \\
$\bar{\Phi}_{2}$ & 1 & $\square$ & $\ldots$ & $\ldots$ & 1 \\
$\vdots$ & $\vdots$ & $\vdots$ & $\vdots$ & $\ddots$ & $\vdots$ \\
$\Phi_{N}$ & 1 & 1 & $\ldots$ & $\ldots$ & $\square$ \\
$\bar{\Phi}_{N}$ & 1 & 1 & $\ldots$ & $\ldots$ & $\bar{\square}$
\end{tabular}


The gauge-invariant Lagrangian for the matter sector can be described by

$$
\begin{array}{r}
\mathcal{L}_{\text {mat }}=\sum_{i=1}^{N}\left[\int d^{2} \theta d^{2} \bar{\theta} K_{h}\left(S, S^{\dagger}\right)\left[\Phi_{i}^{\dagger} e^{2 V_{i}} \Phi_{i}+\bar{\Phi}_{i} e^{-2 V_{i}} \bar{\Phi}_{i}^{\dagger}\right]\right. \\
\left.+\int d^{2} \theta\left[Y \bar{\Phi}_{i} Q_{i} \Phi_{i+1}+Z \bar{\Phi}_{i} \Phi_{i}\right]+\text { h.c. }\right],
\end{array}
$$

where $\Phi_{N+1}\left(\bar{\Phi}_{N+1}\right)$ is identified to $\Phi_{1}\left(\bar{\Phi}_{1}\right)$. We have assumed the coupling constants are universal, for a simplicity. The background chiral superfields $Y$ and $Z$ in the superpotential are the modulus fields providing Yukawa and mass parameters. As will be seen below, these fields are expressed in terms of the moduli $S$ and $Q$. Similar to the vector multiplet case, it is a non-trivial problem to fix the moduli dependence of the normalization function $K_{h}\left(S, S^{\dagger}\right)$. That issue will be discussed later.

\subsubsection{Superpotential}

First we study the moduli dependence of the superpotential terms. It is convenient to rescale the matter multiplets as $(\Phi, \bar{\Phi}) \rightarrow\left\langle K_{h}\right\rangle^{-\frac{1}{2}}(\Phi, \bar{\Phi})$ so that the kinetic terms are canonical. In the rescaled basis, the superpotential contribution to supersymmetric mass terms reads

$$
W_{\text {mass }}=\frac{1}{\left\langle K_{h}\right\rangle} \sum_{i, j} \bar{\Phi}_{i}\left(\langle Y\rangle v \delta_{i+1, j}+\langle Z\rangle \delta_{i, j}\right) \Phi_{j} .
$$

It is easily found that the relation $\langle Y\rangle=\sqrt{2}\left\langle K_{h}\right\rangle$ must be satisfied if one requires the matter spectrum to be equivalent to that of vector multiplet (i.e. that of KK theory in the fivedimensional limit). This implies the moduli form

$$
Y=K_{h}(S, S)
$$

Given this relation, diagonalizing the supersymmetric mass term leads to

$$
W_{\text {mass }}=m_{n} \widetilde{\bar{\Phi}}_{m} \delta_{m n} \widetilde{\Phi}_{n}+\left(\langle Z\rangle /\left\langle K_{h}\right\rangle+v\right) \widetilde{\bar{\Phi}}_{n} \widetilde{\Phi}_{n},
$$

where the supersymmetric eigenstates $\widetilde{\Phi}_{n}$ and $\widetilde{\Phi}_{n}(m, n=0, \cdots, N-1)$ are defined similar to those of vector fields (2.4). The irrelevant phase factors have been absorbed with field redefinitions so that the mass eigenvalues are real. In the absence of nonzero $F$-terms, the mass eigenstates take the same form as the gauge fields. The first term in the right-handed side of (2.20) corresponds to the KK masses and the second one to a bare mass parameter of matter multiplet. When the bare mass $m$ is defined as $\langle Z\rangle /\left\langle K_{h}\right\rangle+v=m / R$, the modulus $Z$ should take the form

$$
Z=\left(\frac{2 \pi m}{N}-1\right) Q K_{h}(S, S)
$$

Now the moduli dependences of the superpotential part are fully determined, their contribution to SUSY-breaking masses can be analyzed. From (2.17), (2.19) and (2.21), we find the mass eigenvalues of spinor components

$$
m_{\psi_{n}}=m_{n}+\frac{m}{R}, \quad(n=0, \cdots, N-1) .
$$


The scalar soft mass terms from the superpotential are written as

$$
\mathcal{L}_{\mathrm{W}}=\left[\left\langle\frac{\partial \ln K_{h}(S, S)}{\partial \ln S}\right\rangle \frac{F_{S}}{\langle S\rangle}+\frac{F_{T}}{\langle T\rangle}\right] \widetilde{\bar{\phi}}_{m}\left(m_{n}+\frac{m}{R}\right) \delta_{m n} \widetilde{\phi}_{n}+\text { h.c. },
$$

where $\phi$ and $\bar{\phi}$ are the scalar components of $\Phi$ and $\bar{\Phi}$, respectively. This is the holomorphic mixing mass term of $\phi$ and $\bar{\phi}$. In the rescaled basis, the mixing masses depend on the Kähler factor $K_{h}$ but they should be cancelled away if one includes full contribution of modulus fields as will be seen below.

\subsubsection{Kähler potential}

Scalar masses also come from the Kähler potential since it has the moduli dependence and so non-canonical. The masses of the spinor components (2.22) does not get changed by the Kähler terms. For the rescaled fields defined above, we read off the Lagrangian (2.17),

$$
\begin{aligned}
\mathcal{L}_{\mathrm{K}}= & \sum_{i=1}^{N}\left[\left|F_{\phi_{i}}\right|^{2}+\left\langle\frac{\partial \ln K_{h}}{\partial S}\right\rangle F_{S} F_{\phi_{i}}^{\dagger} \phi_{i}+\left\langle\frac{\partial \ln K_{h}}{\partial S^{\dagger}}\right\rangle F_{S}^{\dagger} \phi_{i}^{\dagger} F_{\phi_{i}}+\left\langle\frac{1}{K_{h}} \frac{\partial^{2} K_{h}}{\partial S \partial S^{\dagger}}\right\rangle\left|F_{S}\right|^{2} \phi_{i}^{\dagger} \phi_{i}\right. \\
& \left.+\left|F_{\bar{\phi}_{i}}\right|^{2}+\left\langle\frac{\partial \ln K_{h}}{\partial S}\right\rangle F_{S} \bar{\phi}_{i} F_{\bar{\phi}_{i}}^{\dagger}+\left\langle\frac{\partial \ln K_{h}}{\partial S^{\dagger}}\right\rangle F_{S}^{\dagger} F_{\bar{\phi}_{i}} \bar{\phi}_{i}^{\dagger}+\left\langle\frac{1}{K_{h}} \frac{\partial^{2} K_{h}}{\partial S \partial S^{\dagger}}\right\rangle\left|F_{S}\right|^{2} \bar{\phi}_{i} \bar{\phi}_{i}^{\dagger}\right] .
\end{aligned}
$$

Integrating out the matter auxiliary components $F_{\phi_{i}}$ and $F_{\bar{\phi}_{i}}$ and moving to the supersymmetric mass basis, we have

$$
\begin{gathered}
\mathcal{L}_{\mathrm{K}}=-\sum_{n=0}^{N-1}\left[\left(\left|m_{\psi_{n}}\right|^{2}+\left\langle\frac{\partial \ln K_{h}}{\partial S} \frac{\partial \ln K_{h}}{\partial S^{\dagger}}-\frac{1}{K_{h}} \frac{\partial^{2} K_{h}}{\partial S \partial S^{\dagger}}\right\rangle F_{S} F_{S}^{\dagger}\right)\left(\widetilde{\phi}_{n}^{\dagger} \widetilde{\phi}_{n}+\widetilde{\bar{\phi}}_{n} \widetilde{\bar{\phi}}_{n}^{\dagger}\right)\right. \\
\left.+2 m_{\psi_{n}}\left\langle\frac{\partial \ln K_{h}}{\partial S}\right\rangle F_{S} \widetilde{\bar{\phi}}_{n} \widetilde{\phi}_{n}+\text { h.c. }\right] .
\end{gathered}
$$

Combining this with the superpotential contribution to scalar masses (2.23) and noting that $\left\langle\partial \ln K_{h}(S, S) / \partial S\right\rangle=2\left\langle\partial \ln K_{h} / \partial S\right\rangle=2\left\langle\partial \ln K_{h} / \partial S^{\dagger}\right\rangle$, we finally obtain the scalar mass matrix for matter multiplets

$$
\begin{aligned}
& \mathcal{L}_{\text {scalar }}=-\sum_{n=0}^{N-1}\left(\begin{array}{cc}
\widetilde{\phi}_{n}^{\dagger} & \widetilde{\bar{\phi}}_{n}
\end{array}\right)\left(\begin{array}{cc}
\left|m_{\psi_{n}}\right|^{2}+\Gamma\left|\frac{F_{S}}{\langle S\rangle}\right|^{2} & -m_{\psi_{n}}^{*} \frac{F_{T}^{*}}{\langle T\rangle} \\
-m_{\psi_{n}} \frac{F_{T}}{\langle T\rangle} & \left|m_{\psi_{n}}\right|^{2}+\Gamma\left|\frac{F_{S}}{\langle S\rangle}\right|^{2}
\end{array}\right)\left(\begin{array}{c}
\widetilde{\phi}_{n} \\
\overline{\bar{\phi}}_{n}^{\dagger}
\end{array}\right), \\
& \Gamma \equiv\left\langle\frac{\partial^{2} \ln K_{h}^{-1}}{\partial \ln S \partial \ln S^{\dagger}}\right\rangle .
\end{aligned}
$$

Thus the tree-level mass eigenvalues are given by

$$
m_{\phi_{n \pm}}^{2}=\left|m_{\psi_{n}}\right|^{2} \pm m_{\psi_{n}} \frac{F_{T}}{\langle T\rangle}+\Gamma\left|\frac{F_{S}}{\langle S\rangle}\right|^{2}, \quad(n=0, \cdots, N-1) .
$$


One can see in this formula that the effect of the modulus $S$ is controlled by the factor $\Gamma$, which is a function of the normalization constant in the matter Kähler term, while the $F_{T}$ part does not depend on the Kähler normalization. The factor $\Gamma$ becomes, for example, $\Gamma=b / 4$ for the Kähler form

$$
K_{h}=\left(S+S^{\dagger}\right)^{b} X(S) X\left(S^{\dagger}\right),
$$

where $b$ is a constant and $X$ is an arbitrary function. We will find that $b=1$ and $X=$ constant are the appropriate form if one wanted to describe five-dimensional theory. This choice is supported by examining tree-level spectrum and radiative corrections, which will be discussed in the following sections. Such Kähler function indeed satisfies the following nontrivial consistencies that (i) the mass spectrum of matter scalars including SUSY-breaking effects coincides with that of gauginos, (ii) the cutoff dependences of radiative corrections become consistent with known results, and (iii) the moduli dependence of the action has the proper form similar to the argument given at the end of Section 2.1.

\subsubsection{Various limits}

We have determined all the moduli dependences of the action except for $K_{h}$ and presented the superparticle spectrum for vector and matter multiplets in four-dimensions: (2.4), (2.11), (2.12), (2.22), and (2.28). It has been shown for the gaugino mass that several limits of the $F$ terms suggest the five-dimensional properties of SUSY breaking. Let us examine scalar mass spectrum for the typical cases presented in Table 1. The first is the dilaton dominance limit defined by $F_{T}=0$. It is found from (2.28) the scalar mass eigenvalues in this limit become

$$
m_{\phi_{n \pm}}^{2}=\left|m_{\psi_{n}}\right|^{2}+\Gamma\left|\frac{F_{S}}{\langle S\rangle}\right|^{2} \cdot \quad \text { [dilaton] }
$$

As expected, the SUSY-breaking contributions are universal for all scalar fields. Given the Kähler form (2.29) with $b=1$, the spectrum (2.30) (with a vanishing bare mass $m$ ) is identical to that of the gauge fermions (2.13). The second limit we consider is the moduli dominance which leads to the mass eigenvalues

$$
m_{\phi_{n \pm}}^{2}=\left|m_{\psi_{n}}\right|^{2} \pm m_{\psi_{n}} \frac{F_{Q}}{v} . \quad \text { [moduli] }
$$

It is interesting to note that the spectrum is predicted independently of detailed form of the matter Kähler function $K_{h}$. Furthermore the zero mode $(n=0)$ does not get SUSYbreaking effects and remains massless. These features are certainly shared with gauginos. In the approximation that SUSY-breaking effect is much smaller than the compactification scale, the mass eigenvalues of the excited modes is written by $m_{\phi_{n \pm}} \simeq m_{\psi_{n}} \pm F_{Q} / 2 v$, which is also consistent to that of KK gauginos.

In the limit $F_{S 5}=0$, we have

$$
m_{\phi_{n \pm}}^{2}=\left|m_{\psi_{n}}\right|^{2} \pm m_{\psi_{n}} \frac{F_{T}}{\langle T\rangle}+\Gamma\left|\frac{F_{T}}{\langle T\rangle}\right|^{2} . \quad \text { [radion] }
$$


The mass eigenvalues (2.32) have the same form as those of gauginos when $b=1$ for the matter Kähler form. Note that the masses of the excited modes approximately agree with the moduli dominance limit. The only difference is whether the zero mode is massless or not, which mode is identified to the low-energy degree of freedom in the five-dimensional viewpoint.

We thus find from the tree-level analysis of mass spectrum that $b=1$ in the Kähler form (2.29) is a suitable choice for the normalization of matter multiplets. The remaining functional dependence $X(S)$ will be fixed by loop-level consistency of theory.

\section{$2.3 \quad$ Orbifolding}

The analysis above has been performed for the case that corresponds to the circle compactification of the fifth dimension. It is straightforward to extend it to a compactification on the line segment. For vector multiplets, what we have to do is removing a bi-fundamental field, e.g. $Q_{N}$. This procedure leads to only a four-dimensional vector multiplet as the light degrees of freedom. The gauge anomaly arising from removing $Q_{N}$ can be supplemented by introducing appropriate chiral fields which are charged under the $G_{1}$ and $G_{N}$ gauge symmetries. Effects of these 'local' fields can be neglected in the large $N$ limit. For matter multiplets, the absence of one anti-chiral multiplet, e.g. $\bar{\Phi}_{N}$, leaves a chiral zero mode of the fundamental representation of the diagonal subgroup $G$. If one chiral multiplet, e.g. $\Phi_{1}$, is removed, a zero mode is anti-fundamental representation. In these cases, suitable anomaly cancellations are also required. The gauge-invariant Lagrangian and its moduli dependences are almost same as before. For example, when $\bar{\Phi}_{N}$ is removed, the mass eigenvalues and eigenstates are given by

$$
\begin{aligned}
& m_{n}=m_{\psi_{n}}=2 v \sin \left(\frac{n \pi}{2 N}\right) \quad(n=0, \cdots, N-1), \\
& \widetilde{\Phi}_{n}=\sqrt{\frac{2}{2^{\delta_{n 0}} N}} \sum_{j=1}^{N} \cos \left(\frac{2 j-1}{2 N} n \pi\right) \Phi_{j}, \quad \widetilde{\bar{\Phi}}_{n}=\sqrt{\frac{2}{N}} \sum_{j=1}^{N-1} \sin \left(\frac{j}{N} n \pi\right) \bar{\Phi}_{j} .
\end{aligned}
$$

The result can be obtained for the case of removing $\Phi_{1}$ by exchanging $\Phi \leftrightarrow \bar{\Phi}$, and in a similar way for vector multiplets. The SUSY-breaking mass formulas do not change except for the expression of $m_{n}$. It is, however, noted as a difference from the previous section that bare mass parameters of matter multiplets cannot be introduced unless there is a set of matter multiplets which leaves vector-like massless modes.

\section{Quantum analysis}

We have discussed SUSY-breaking effects through the moduli $S$ and $Q$, and calculated the tree-level spectrum by determining the proper moduli dependences of the action. We have particularly shown that on the specific lines in the parameter space of $F_{S}$ and $F_{Q}$, the sparticle spectra are consistent to the SUSY-breaking patterns in higher dimensions. In some cases, low-energy degrees of freedom do not receive SUSY-breaking effects and remain massless in the tree-level approximation. It is then important, e.g. for realistic model building, to include radiative corrections. In this section, we will calculate various types of one-loop corrections to 
gaugino and Higgs scalar masses from gauge and Yukawa interactions. In the following, the analysis will be performed in the case of the orbifold compactification.

\subsection{Gaugino masses}

\subsubsection{Vector contribution}

The first type of one-loop corrections which contribute to zero-mode gaugino masses involves vector and adjoint scalar multiplets running in the loops. Let us recall that the mass matrix of the $n$-th massive gauge fermions takes the form:

$$
-\frac{1}{2}\left(\begin{array}{cc}
\tilde{\lambda}_{n} & \tilde{\chi}_{n}
\end{array}\right)\left(\begin{array}{cc}
\frac{F_{S}}{2\langle S\rangle} & m_{n} \\
m_{n} & -\frac{F_{Q}}{\langle Q\rangle}-\frac{F_{S}}{2\langle S\rangle}
\end{array}\right)\left(\begin{array}{c}
\tilde{\lambda}_{n} \\
\tilde{\chi}_{n}
\end{array}\right)+\text { h.c. }
$$

where $\chi_{n}$ are the goldstone fermions associated with the gauge symmetry breaking. The eigenvalues $m_{\lambda_{n \pm}}$ of this matrix were given by (2.11) and the mixing angle between $\lambda_{n}$ and $\chi_{n}$ upon the diagonalization satisfies

$$
\tan 2 \theta_{n}=\frac{2 m_{n}\left\langle S_{5}\right\rangle}{F_{S 5}}
$$

At the component level, there are two types of one-loop diagrams for the gaugino two-point function, which come from gauge fields and adjoint scalars, respectively,

$$
\begin{aligned}
I_{\text {gauge }} & =\left(\frac{g}{\sqrt{N}}\right)^{2} C_{2}(G) \sum_{n=0}^{N-1} \int \frac{d^{4} k}{i(2 \pi)^{4}} \frac{g_{\mu \nu}}{(p-k)^{2}-m_{n}^{2}} \sigma^{\mu}\left[\frac{\cos ^{2} \theta_{n}}{m_{\lambda_{n+}}-\not k}+\frac{\sin ^{2} \theta_{n}}{m_{\lambda_{n-}}-\not k}\right] \sigma^{\nu}, \\
I_{\text {adj }} & =\left(\frac{\sqrt{2} g}{\sqrt{N}}\right)^{2} C_{2}(G) \sum_{n=0}^{N-1} \int \frac{d^{4} k}{i(2 \pi)^{4}} \frac{1}{m_{c_{n}}^{2}-(p-k)^{2}}\left[\frac{\sin ^{2} \theta_{n}}{m_{\lambda_{n+}}-\not k}+\frac{\cos ^{2} \theta_{n}}{m_{\lambda_{n-}}-\not k}\right],
\end{aligned}
$$

where $p$ is the four-momentum of the external gaugino line and $C_{2}(G)$ is the quadratic Casimir for the adjoint representation of the gauge group $G$. With the introduction of a ultraviolet cutoff $\Lambda$, the divergence parts are calculated as

$$
\begin{aligned}
I_{\text {gauge }}^{\text {div }} & =\frac{C_{2}(G)}{16 \pi^{2}}\left(\frac{g}{\sqrt{N}}\right)^{2} \sum_{n=0}^{N-1}\left(\not p-4\left(m_{\lambda_{n+}} \cos ^{2} \theta_{n}+m_{\lambda_{n-}} \sin ^{2} \theta_{n}\right)\right) \ln \Lambda^{2}, \\
I_{\text {adj }}^{\text {div }} & =\frac{C_{2}(G)}{16 \pi^{2}}\left(\frac{g}{\sqrt{N}}\right)^{2} \sum_{n=0}^{N-1}\left(\not p+2\left(m_{\lambda_{n+}} \sin ^{2} \theta_{n}+m_{\lambda_{n-}} \cos ^{2} \theta_{n}\right)\right) \ln \Lambda^{2} .
\end{aligned}
$$

These divergences should be renormalized by appropriate counter terms. Note in particular that the total one-loop divergent correction to gaugino mass becomes

$$
\delta m^{(1)}=\frac{2 N C_{2}(G)}{16 \pi^{2}}\left(\frac{g}{\sqrt{N}}\right)^{2} \frac{F_{S 5}}{\left\langle S_{5}\right\rangle} \ln \Lambda^{2} .
$$

It is interesting that since this total mass divergence is proportional to $F_{S 5}$, there appears no

ultraviolet divergence in the limit of the radius modulus SUSY breaking. This is indeed a 
consistent result and will be discussed in detail later on. On the other hand, the divergences generally appear in other cases, for example, in the dilaton/moduli dominant cases.

The finite part of the gauge field correction $I_{\text {gauge }}$ is given by

$$
I_{\text {gauge }}^{\text {fin }}=\frac{-2 N C_{2}(G)}{16 \pi^{2}}\left(\frac{g}{\sqrt{N}}\right)^{2} \frac{F_{T}}{\langle T\rangle} .
$$

We have used an approximation that the zero-mode gaugino mass, i.e. the SUSY-breaking mass scale is much smaller than KK masses. The finite correction from $I_{\text {adj }}$ can also be estimated in a similar way and is found to be $-\frac{1}{2} I_{\text {gauge }}^{\text {fin }}$. We thus obtain the zero-mode gaugino mass up to one-loop level,

$$
m_{\lambda_{0 \pm}}= \pm \frac{F_{S 5}}{2\left\langle S_{5}\right\rangle}-\frac{F_{T}}{2\langle T\rangle}-\frac{2 N C_{2}(G) g_{4}^{2}}{16 \pi^{2}} \frac{F_{T}}{\langle T\rangle} .
$$

The four-dimensional effective gauge coupling $g_{4}$ is defined in (2.8). One can find two important points from this expression of one-loop gaugino masses. Firstly, the radiative correction is proportional to $F_{T}$ but not $F_{S}$. This fact seems to agree with a result of the string-inspired supergravity models [10]. There one-loop corrections to gaugino masses arise from string threshold corrections or effects of bulk fields, and are specified by the modulus field $T$. The second point is that the size of one-loop correction is controlled by the gauge beta function of heavy fields. This fact coincides with the spectrum of Ref. [13] which is determined by wavefunction renormalizations from vector messengers [14]. The dependence on beta-function coefficients also appears in the supergravity models.

\subsubsection{Matter contribution}

Consider one-loop contribution to gaugino masses from matter multiplets. With the mass splitting between matter fermions and sfermions, the one-loop correction to the gaugino twopoint function is evaluated as

$$
\begin{aligned}
I_{\mathrm{mat}}=\left(\frac{\sqrt{2} g}{\sqrt{N}}\right)^{2} T_{2}(R) \sum_{n=0}^{N-1} \int \frac{d^{4} k}{i(2 \pi)^{4}}\left[\frac{1}{m_{n}-\not k}\left(\frac{1 / 2}{m_{\phi_{n+}}^{2}-(p-k)^{2}}+\frac{1 / 2}{m_{\phi_{n-}}^{2}-(p-k)^{2}}\right)\right. \\
\left.-\frac{1}{m_{n}+\not k}\left(\frac{1 / 2}{m_{\phi_{n+}}^{2}-(p-k)^{2}}+\frac{1 / 2}{m_{\phi_{n-}}^{2}-(p-k)^{2}}\right)\right]
\end{aligned}
$$

where $T_{2}(R)$ is the quadratic Dynkin index for the representation $R$ of the unbroken gauge group $G$. The scalar mass eigenvalues $m_{\phi_{n \pm}}$ are given in Eq. (2.28) with $\Gamma=1 / 4$ (see the discussions in Sections 2.2.2 and 2.2.3). The divergent part of (3.10) is given by

$$
I_{\mathrm{mat}}^{\mathrm{div}}=\frac{2 N T_{2}(R)}{16 \pi^{2}}\left(\frac{g}{\sqrt{N}}\right)^{2} \not p \ln \Lambda^{2} .
$$

Note that this is a supersymmetric correction. There is no divergence to gaugino masses from matter multiplets since only scalar components receive SUSY-breaking masses. In other 
words, the loop integrals converge if the scalar propagators are expanded with respect to SUSY-breaking VEVs. As for the finite part, we obtain

$$
I_{\mathrm{mat}}^{\mathrm{fin}}=\frac{N T_{2}(R)}{16 \pi^{2}}\left(\frac{g}{\sqrt{N}}\right)^{2} \frac{F_{T}}{\langle T\rangle} .
$$

One can see that as in the vector case, the matter contribution is controlled by the modulus $T$. After all, the total amount of mass corrections is read from (3.9) and (3.12), and the gaugino mass up to one-loop level is given by

$$
m_{\lambda_{0 \pm}}= \pm \frac{F_{S 5}}{2\left\langle S_{5}\right\rangle}-\frac{F_{T}}{2\langle T\rangle}+N\left[-2 C_{2}(G)+2 T_{2}(R)\right] \frac{g_{4}^{2}}{16 \pi^{2}} \frac{F_{T}}{\langle T\rangle} .
$$

The radiative correction is proportional to $F_{T}$ and the gauge beta function of heavy supermultiplets.

\section{$3.2 \quad$ Higgs masses}

Next we study one-loop radiative corrections to masses of Higgs scalars. Having the large top Yukawa coupling in mind, the corrections from various types of large Yukawa couplings will be investigate in detail. Gauge corrections can be estimated in a similar way and give qualitatively similar results. In the numerical evaluations, we will focus on the three special limits discussed in Section 2, and find that all types of radiative corrections to Higgs masses are consistent with the expected SUSY-breaking patterns. In particular, the corrections become ultraviolet finite in the limit of the radius modulus SUSY breaking. (We have already found in the previous section the same result for gaugino masses [see (3.7)].) The compactificationscale dependence of radiative corrections is another important factor to be examined. We will show that its quantitative behavior is also properly taken into account in our framework.

\subsubsection{Bulk-Brane-Brane couplings}

Let us study the radiative corrections from Yukawa couplings involving KK modes of matter fields. The first case we consider is a Yukawa coupling among one 'bulk' field and two 'brane' fields. Suppose that $L_{i}(i=1, \cdots, N)$ and $\bar{L}_{j}(j=1, \cdots, N-1)$ are introduced as bulk matter multiplets as described in Section 2.2 and $e$ as a chiral superfield charged only under $G_{1}$ (wouldbe a localized field on a brane in the five-dimensional point of view). The transformation properties are listed below;

\begin{tabular}{c|ccccc} 
& $G_{1}$ & $G_{2}$ & $G_{3}$ & $\ldots$ & $G_{N}$ \\
\hline$L_{1}$ & $\square$ & 1 & $\cdots$ & $\cdots$ & 1 \\
$\bar{L}_{1}$ & $\square$ & 1 & $\ldots$ & $\ldots$ & 1 \\
$L_{2}$ & 1 & $\square$ & $\ldots$ & $\ldots$ & 1 \\
$\bar{L}_{2}$ & 1 & $\bar{\square}$ & $\ldots$ & $\ldots$ & 1 \\
$\vdots$ & $\vdots$ & $\vdots$ & $\vdots$ & $\ddots$ & $\vdots$ \\
$e$ & $\square$ & 1 & $\ldots$ & $\ldots$ & 1 \\
$H$ & 1 & 1 & $\ldots$ & $\ldots$ & 1
\end{tabular}


In addition to the tree-level matter Lagrangian (2.17), we have a gauge-invariant Yukawa term among $L_{1}, e$ and the Higgs field $H$. Other gauge invariant couplings may be forbidden by symmetry arguments, for example, an invariance under $e \rightarrow-e$ and $H \rightarrow-H$. The resultant superpotential is written in terms of the mass eigenstates of $L$ 's defined in Section 2;

$$
W=y\left(\sum_{n=0}^{N-1} \eta_{n}^{L} \widetilde{L}_{n}\right) e H+\sum_{n=0}^{N-1} m_{L_{n}} \widetilde{L}_{n} \widetilde{\bar{L}}_{n}
$$

where $m_{L_{n}}$ are the KK masses (2.22), and $\eta_{n}^{L}$ denotes the mixing between $L_{1}$ and the mass eigenstate $\widetilde{L}_{n}$. Its explicit form has been derived in Section 2.3:

$$
\eta_{n}^{L}=\sqrt{\frac{2}{2^{\delta_{n 0} N}}} \cos \left(\frac{n \pi}{2 N}\right) .
$$

Eliminating the matter auxiliary fields from the Lagrangian (2.17) and (3.15) leads to

$$
\begin{aligned}
\mathcal{L}=-y \psi_{e} & \left(\sum_{n=0}^{N-1} \eta_{n}^{L} \psi_{\widetilde{L}_{n}}\right) H-\frac{|y|^{2}}{2}\left(\left|\sum_{n=0}^{N-1} \eta_{n}^{L} \widetilde{L}_{n}^{\prime}\right|^{2}+\left|\sum_{n=0}^{N-1} \eta_{n}^{L *} \widetilde{\bar{L}}_{n}^{\prime}\right|^{2}\right) H^{\dagger} H \\
& -\frac{y}{\sqrt{2}} e H \sum_{n=0}^{N-1} \eta_{n}^{L}\left[\left(\frac{F_{S}}{2\langle S\rangle}-m_{L_{n}}^{*}\right) \widetilde{L}_{n}^{\prime}+\left(\frac{F_{S}}{2\langle S\rangle}+m_{L_{n}}^{*}\right) \widetilde{\bar{L}}_{n}^{\prime}\right]+\text { h.c. }+\cdots
\end{aligned}
$$

where $\psi_{X}$ means the spinor component of a chiral multiplet $X$. The sfermion fields with primes are the mass eigenstates which completely diagonalize the mass matrix involving SUSYbreaking effects,

$$
\widetilde{L}_{n}=\frac{1}{\sqrt{2}} \widetilde{L}_{n}^{\prime}+\frac{1}{\sqrt{2}} \widetilde{\bar{L}}_{n}^{\prime}, \quad \widetilde{\bar{L}}_{n}=\frac{-1}{\sqrt{2}} \widetilde{L}_{n}^{\prime}+\frac{1}{\sqrt{2}} \widetilde{\bar{L}}_{n}^{\prime} .
$$

It is important to notice that in deriving the Lagrangian (3.17), we have fixed the normalization function $K_{h}$ for matter multiplets as $\left\langle\frac{\partial \ln K_{h}}{\partial S}\right\rangle=\frac{1}{2\langle S\rangle}$. As will be seen below, this form of VEV is required for the model to be consistent at a quantum level. Together with Eq. (2.29), we obtain

$$
K_{h}\left(S, S^{\dagger}\right)=c\left(S+S^{\dagger}\right)
$$

with an arbitrary constant $c$.

With these matter-Higgs interactions at hand, we have three types of diagrams which contribute to the Higgs mass corrections. Each diagram involves an interaction in the Lagrangian (3.17): (1) Yukawa couplings with the fermions running in the loop, (2) sfermion-Higgs quartic couplings, and (3) sfermion-Higgs trilinear couplings. These contributions to the Higgs two-point function become

$$
\begin{aligned}
I^{(1)} & =-2|y|^{2} \sum_{n=0}^{N-1} \int \frac{d^{4} p}{i(2 \pi)^{4}} \frac{\left|\eta_{n}^{L}\right|^{2}}{p^{2}-m_{L_{n}}^{2}}, \\
I^{(2)} & =|y|^{2} \sum_{n=0}^{N-1} \int \frac{d^{4} p}{i(2 \pi)^{4}} \frac{\left|\eta_{n}^{L}\right|^{2}}{2}\left[\frac{1}{p^{2}-m_{L_{n+}}^{2}}+\frac{1}{p^{2}-m_{L_{n-}}^{2}}\right]+|y|^{2} \int \frac{d^{4} p}{i(2 \pi)^{4}} \frac{1}{p^{2}},
\end{aligned}
$$




$$
I^{(3)}=|y|^{2} \sum_{n=0}^{N-1} \int \frac{d^{4} p}{i(2 \pi)^{4}} \frac{\left|\eta_{n}^{L}\right|^{2}}{2}\left[\frac{\left|\frac{F_{S}}{2\langle S\rangle}-m_{L_{n}}^{*}\right|^{2}}{p^{2}-m_{L_{n+}}^{2}}+\frac{\left|\frac{F_{S}}{2\langle S\rangle}+m_{L_{n}}^{*}\right|^{2}}{p^{2}-m_{L_{n-}}^{2}}\right] \frac{1}{p^{2}} .
$$

The sfermion mass eigenvalues $m_{L_{n \pm}}^{2}$ are given by (2.28). We show in Fig. 1 the total one-loop correction $I=I^{(1)}+I^{(2)}+I^{(3)}$. The horizontal axis denotes the momentum cutoff $\Lambda$ normalized by the compactification scale. We particularly focus on the three limits of the SUSY-breaking order parameters which were presented in Table 1. It is found from the figure that the loop

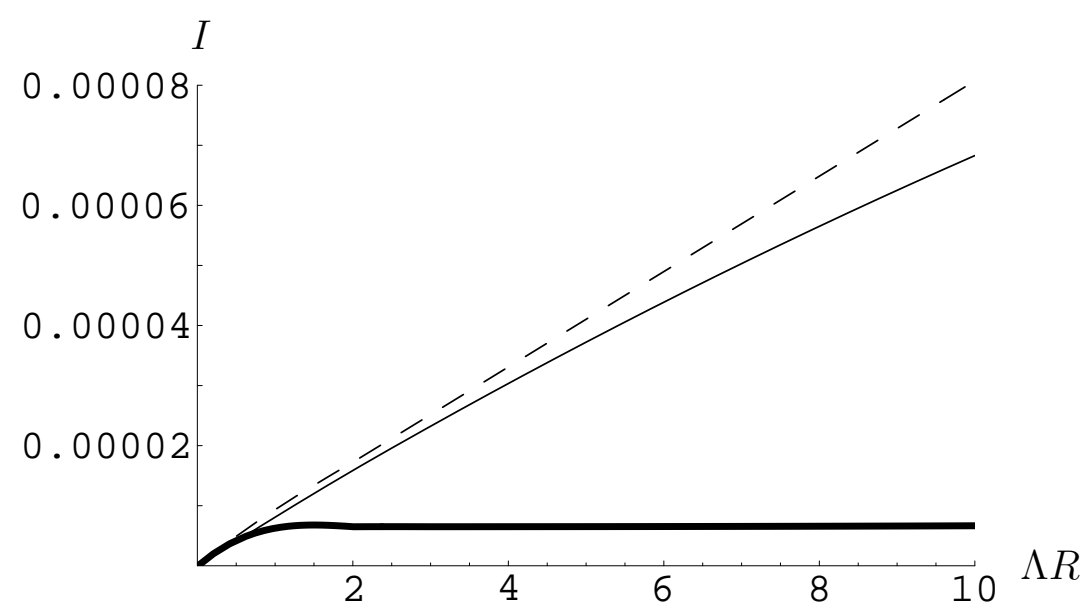

Figure 1: The ultraviolet cutoff dependences of the loop correction from the bulk-brane-brane type Yukawa coupling. From the upper, the dilaton, moduli and the radius modulus SUSY breaking are plotted. We take $y=1$ and $N=200$ as an example.

correction is independent of the momentum cutoff and becomes finite in the limit of the radius modulus breaking (bold line). On the other hand, in the dilaton/moduli dominance scenarios (dashed/solid lines), the corrections linearly depend on $\Lambda$. This behavior is understood as the number of KK modes running around the loop. That is, the KK modes whose masses are below the momentum cutoff can contribute to the radiative corrections. In these three limited cases, the structures of radiative corrections are consistent with the results which have been obtained in the literature.

The finite result for the Higgs mass is analytically understood by noting that, in the limit $F_{S 5} /\left\langle S_{5}\right\rangle=F_{S} /\langle S\rangle+F_{T} /\langle T\rangle=0$, the total correction $I$ is simply summarized as

$$
I\left(F_{S_{5}}=0\right)=-|y|^{2} \sum_{n=0}^{N-1} \sum_{i=+,-} \int \frac{d^{4} p}{i(2 \pi)^{4}}\left|\eta_{n}^{L}\right|^{2}\left[\frac{1}{p^{2}-m_{L_{n}}^{2}}-\frac{1}{p^{2}-m_{L_{n i}}^{2}}\right] .
$$

The first term in the integrand represents the fermionic contribution and the second one the bosonic contribution. The ultraviolet finiteness of this type of momentum integral will be discussed in Section 5. 


\subsubsection{Bulk-Bulk-Brane couplings}

In a similar way, we calculate one-loop Higgs masses from bulk-bulk-brane type Yukawa couplings. In this case, the matter multiplets $L_{i}\left(\bar{L}_{i}\right)$ and $e_{j}\left(\bar{e}_{j}\right)$ are charged under the $G$ gauge symmetry, and the Higgs $H$ is introduced as a singlet field. To implement the orbifold projection, $L_{N}$ and $\bar{e}_{1}$ are removed from the spectrum. The transformation properties of these fields are given by

\begin{tabular}{c|ccccc} 
& $G_{1}$ & $G_{2}$ & $G_{3}$ & $\cdots$ & $G_{N}$ \\
\hline$L_{1}$ & $\square$ & 1 & $\cdots$ & $\cdots$ & 1 \\
$\bar{L}_{1}$ & $\bar{\square}$ & 1 & $\cdots$ & $\cdots$ & 1 \\
$L_{2}$ & 1 & $\square$ & $\cdots$ & $\cdots$ & 1 \\
$\bar{L}_{2}$ & 1 & $\bar{\square}$ & $\cdots$ & $\cdots$ & 1 \\
$\vdots$ & $\vdots$ & $\vdots$ & $\vdots$ & $\vdots$ & $\vdots$ \\
$e_{1}$ & $\bar{\square}$ & 1 & $\cdots$ & $\cdots$ & 1 \\
$e_{2}$ & 1 & $\bar{\square}$ & $\cdots$ & $\cdots$ & 1 \\
$\bar{e}_{2}$ & 1 & $\square$ & $\cdots$ & $\cdots$ & 1 \\
$\vdots$ & $\vdots$ & $\vdots$ & $\vdots$ & $\vdots$ & $\vdots$ \\
$H$ & 1 & 1 & $\cdots$ & $\cdots$ & 1
\end{tabular}

The superpotential terms for masses and Yukawa couplings are written in the mass eigenstate basis defined in a similar fashion to (3.18),

$$
W=y\left(\sum_{n=0}^{N-1} \eta_{n}^{L} \widetilde{L}_{n}\right)\left(\sum_{l=0}^{N-1} \eta_{l}^{e} \widetilde{e}_{l}\right) H+\sum_{n=0}^{N-1} m_{L_{n}} \widetilde{L}_{n} \widetilde{\bar{L}}_{n}+\sum_{n=0}^{N-1} m_{e_{n}} \widetilde{e}_{n} \widetilde{\bar{e}}_{n} .
$$

Notice that we have introduced only one type of Yukawa term $y L_{1} e_{1} H$. This is analogous to the fact that a bulk Yukawa coupling is not consistent with symmetries of five-dimensional supersymmetric theories. In the present four-dimensional model, other gauge-invariant Yukawa terms can be forbidden by some discrete symmetry, as already done for the bulk-brane-brane type Yukawa terms. Integrating out the matter multiplet $F$ terms, we obtain the following interaction terms

$$
\begin{aligned}
\mathcal{L}=- & \frac{|y|^{2}}{2}\left[\left|\sum_{n=0}^{N-1} \eta_{n}^{L} \widetilde{L}_{n}^{\prime}\right|^{2}+\left|\sum_{n=0}^{N-1} \eta_{n}^{L *} \widetilde{\bar{L}}_{n}^{\prime}\right|^{2}+\left|\sum_{n=0}^{N-1} \eta_{n}^{e} \widetilde{e}_{n}\right|^{2}+\left|\sum_{n=0}^{N-1} \eta_{n}^{e *} \widetilde{\bar{e}}_{n}^{\prime}\right|^{2}\right] H^{\dagger} H \\
& -\frac{y}{2} H \sum_{n=0}^{N-1} \sum_{l=0}^{N-1} \eta_{n}^{e} \eta_{l}^{L}\left(\widetilde{e}_{n}^{\prime}-\widetilde{\bar{e}}_{n}^{\prime \dagger}\right)\left[\left(\frac{F_{S}}{2\langle S\rangle}-m_{L_{l}}^{*}\right) \widetilde{L}_{l}^{\prime}+\left(\frac{F_{S}}{2\langle S\rangle}+m_{L_{l}}^{*}\right) \widetilde{\bar{L}}_{l}^{\prime}\right] \\
& -\frac{y}{2} H \sum_{n=0}^{N-1} \sum_{l=0}^{N-1} \eta_{n}^{L} \eta_{l}^{e}\left(\widetilde{L}_{n}^{\prime}-\widetilde{\bar{L}}_{n}^{\prime \dagger}\right)\left[\left(\frac{F_{S}}{2\langle S\rangle}-m_{e_{l}}^{*}\right) \widetilde{e}_{l}^{\prime}+\left(\frac{F_{S}}{2\langle S\rangle}+m_{e_{l}}^{*}\right) \widetilde{\bar{e}}_{l}^{\dagger}\right] \\
& -y\left(\sum_{n=0}^{N-1} \eta_{n}^{e} \psi_{\widetilde{e}_{n}}\right)\left(\sum_{l=0}^{N-1} \eta_{l}^{L} \psi_{\widetilde{L}_{l}}\right) H+\text { h.c. }+\cdots
\end{aligned}
$$

With this Lagrangian, the diagrams of loop corrections to the Higgs mass are similar to those in the previous section: (1) Yukawa couplings to the Higgs, (2) sfermion-Higgs quartic couplings, 
and (3) sfermion-Higgs trilinear couplings. Each contribution is given by

$$
\begin{aligned}
I^{(1)}= & -2|y|^{2} \sum_{n=0}^{N-1} \sum_{l=0}^{N-1} \int \frac{d^{4} p}{i(2 \pi)^{4}}\left|\eta_{n}^{L}\right|^{2}\left|\eta_{l}^{e}\right|^{2} \frac{p^{2}}{\left(p-m_{L_{n}}\right)\left(p-m_{e_{l}}\right)}, \\
I^{(2)=} & |y|^{2} \sum_{n=0}^{N-1} \int \frac{d^{4} p}{i(2 \pi)^{4}} \frac{\left|\eta_{n}^{L}\right|^{2}}{2}\left[\frac{1}{p^{2}-m_{L_{n+}}^{2}}+\frac{1}{p^{2}-m_{L_{n-}}^{2}}\right]+(L \leftrightarrow e), \\
I^{(3)=} & |y|^{2} \sum_{n=0}^{N-1} \sum_{l=0}^{N-1} \int \frac{d^{4} p}{i(2 \pi)^{4}} \frac{\left|\eta_{n}^{L}\right|^{2}}{2} \frac{\left|\eta_{l}^{e}\right|^{2}}{2}\left[\frac{1}{p^{2}-m_{e_{n+}}^{2}}+\frac{1}{p^{2}-m_{e_{n-}}^{2}}\right] \\
& \times\left[\frac{\left|\frac{F_{S}}{2\langle S\rangle}-m_{L_{n}}^{*}\right|^{2}}{p^{2}-m_{L_{n+}}^{2}}+\frac{\left|\frac{F_{S}}{2\langle S\rangle}+m_{L_{n}}^{*}\right|^{2}}{p^{2}-m_{L_{n-}}^{2}}\right]+(L \leftrightarrow e) .
\end{aligned}
$$

The total loop correction $I=I^{(1)}+I^{(2)}+I^{(3)}$ is plotted for several cases in Fig. 2, where the Yukawa coupling constant $y$ is taken to be 1, as an example. One can see that the

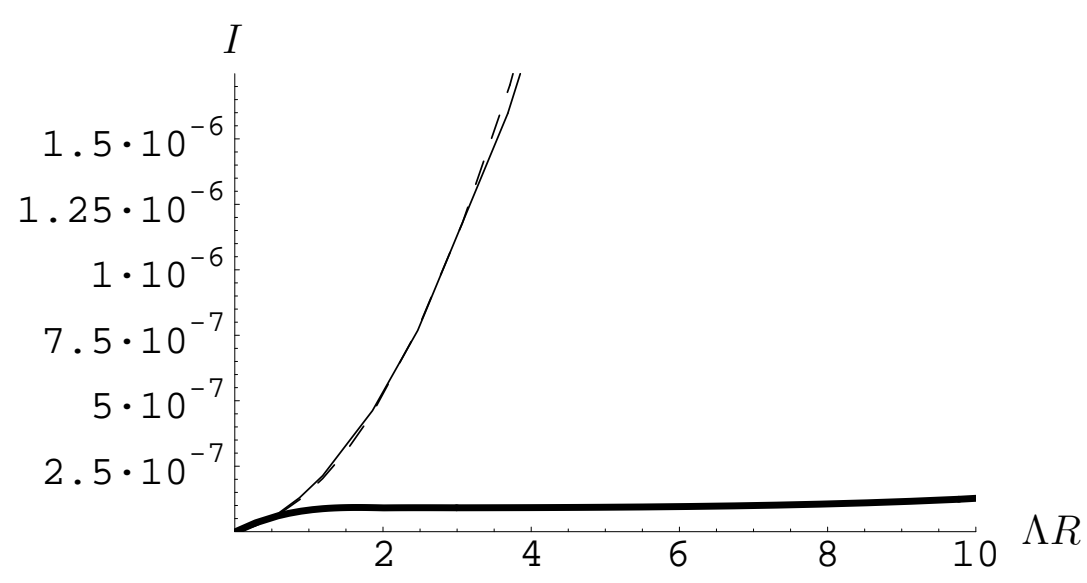

Figure 2: The cutoff dependences of the loop correction from the bulk-bulk-brane type Yukawa coupling. The dilaton, moduli and radius modulus SUSY breaking are plotted from the above (the dashed, solid, and bold lines, respectively). The parameters are same as in Figure 1.

cutoff dependences of the loop correction have similar behaviors as in the previous section; $I$ is insensitive to the momentum cutoff $\Lambda$ for the radius modulus SUSY breaking, while $I$ is cutoff-dependent (roughly proportional to $\Lambda^{2}$ ) for the other cases, which dependence is interpreted as the number of KK modes circulating in the loops.

\subsubsection{Bulk-Bulk-Bulk couplings}

Finally we consider the case that all $L, e$ and $H$ are introduced as bulk multiplets, and examine radiative corrections to the massless Higgs scalar. Suppose that corrections come from the superpotential Yukawa coupling $L_{1} e_{1} H_{1}$ and other Yukawa terms are absent. Thus 
the situation seems to be similar to the bulk-bulk-brane Yukawa case in Section 3.2.2, except for an overall rescaling of Yukawa couplings. However we now have an additional scalar 3-point vertex which is a cross term generated by integrating out the $F$ component of Higgs multiplet,

$$
-y \sum_{n=0}^{N-1} \eta_{n}^{H}\left(\left\langle\frac{\partial \ln K_{h}}{\partial \ln S}\right\rangle \frac{F_{S}}{\langle S\rangle} \widetilde{H}_{n}+m_{H_{n}}^{*} \widetilde{\bar{H}}_{n}^{\dagger}\right)\left(\sum_{n=0}^{N-1} \eta_{n}^{L} \widetilde{L}_{n}\right)\left(\sum_{l=0}^{N-1} \eta_{l}^{e} \widetilde{e}_{l}\right)+\text { h.c.. }
$$

Other couplings of the Higgs zero mode are modified only by the rescaling with the wavefunctions $\eta_{0}^{H, \bar{H}}=1 / \sqrt{N}$, which does not cause any qualitative changes to mass corrections.

While the corrections induced by the vertex (3.30) are generally divergent logarithmically, no qualitative change is found for the supergravity SUSY-breaking models, compared to the bulk-bulk-brane Yukawa case. On the other hand, one might wonder that the vertex (3.30) gives rise to a cutoff-dependent contribution for the radius modulus breaking where even logarithmic divergences are vanishing. However, it is found from the matter Kähler form (3.19) that the vertex (3.30) leads to the interactions of the Higgs $n=0$ modes

$$
-\frac{y}{\sqrt{2 N}}\left(m_{H_{0+}} \widetilde{H}_{0}^{\prime}+m_{H_{0-}}{\widetilde{\bar{H}_{0}^{\prime}}}^{\dagger}\right)\left(\sum_{n=0}^{N-1} \eta_{n}^{L} \widetilde{L}_{n}\right)\left(\sum_{l=0}^{N-1} \eta_{l}^{e} \widetilde{e}_{l}\right)+\text { h.c. },
$$

in the $F_{S 5}=0$ limit. Therefore the coupling of the Higgs massless mode of (3.30) is vanishing, independently of which of the Higgs fields $H$ and $\bar{H}$ contain the zero mode. As a result, the radiative corrections from bulk-bulk-bulk Yukawa couplings are qualitatively unchanged from the bulk-bulk-brane Yukawa case in the previous section.

\subsection{Radius dependence}

We have analyzed the momentum cutoff dependence of the radiative corrections. As another important factor, let us examine how the loop corrections to scalar masses depend on the compactification radius $R$ of the fifth dimension. Consider for example the correction from a bulk-brane-brane type Yukawa coupling. When all the parameters except for $R$ are fixed, the following $R$ dependences of one-loop corrections to scalar masses are expected;

$$
\begin{array}{rlll}
\delta m_{\phi}^{2} & \propto R & \text { [dilaton] } \\
\delta m_{\phi}^{2} & \propto R^{3} & \text { [moduli], } \\
\delta m_{\phi}^{2} & \propto R^{-2} & \text { [radion]. }
\end{array}
$$

These behaviors can be understood as follows. In the dilaton dominant case, the $R^{1}$ behavior is interpreted as the number of KK modes $(\simeq \Lambda R)$ propagating in loop diagrams. In the moduli dominant case, we have an $R^{3}$ factor from the number of KK modes $\left(R^{1}\right)$ and SUSY-breaking effects $\left[\left(F_{T} /\langle T\rangle\right)^{2} \propto R^{2}\right]$. On the other hand, the radiative corrections to Higgs masses are expected to behave like $R^{-2}$ in the radius modulus breaking. This is because the radius modulus breaking is equivalent to the boundary condition breaking of supersymmetry [16] and hence quadratic divergences are cut off by the compactification scale due to the locality in the extra dimension. 
In Fig. 3, we show the radius dependences of the scalar mass correction from bulk-branebrane Yukawa couplings (Section 3.2.1). The horizontal axis denotes the size of $R$ and the vertical one the magnitudes of one-loop corrections. In the figure, $R$ is taken to be within the regime $1<\Lambda R<10$ ( $\Lambda$ is the momentum cutoff). The upper bound comes from the fact that finite scalar masses are realized in the region $\Lambda \ll v$ and the lower bound from the requirement that at least one KK mode runs around the loop. One can see from the figure that the expected results (3.32)-(3.34) are certainly reproduced in our model.

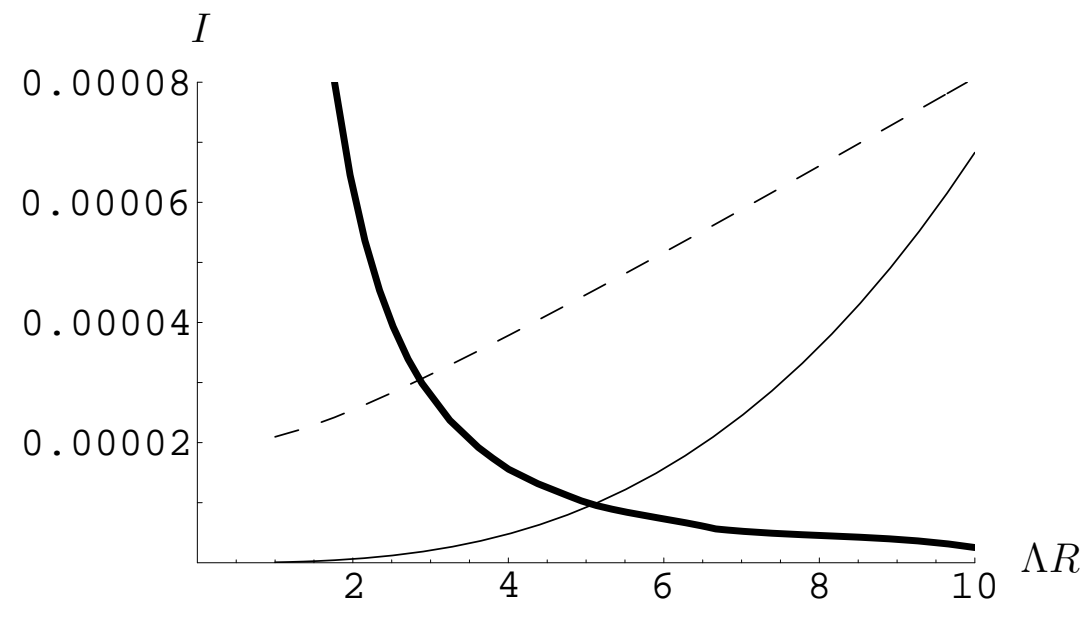

Figure 3: The radius dependences of the radiative correction to the Higgs mass. The horizontal axis means the compactification radius (normalized by the ultraviolet cutoff) and the vertical one the magnitudes of the correction. The dashed, solid, and bold lines correspond to the dilaton dominance, the moduli dominance, and the radius modulus breaking, respectively. The parameters are same as in Figure 1.

Summarizing Section 3, we have explicitly calculated one-loop radiative corrections to gaugino and Higgs scalar masses. The one-loop gaugino masses are proportional to the gauge beta function of heavy fields and also to the modulus auxiliary $\mathrm{VEV} F_{T}$. This seems to be a consistent result with the predictions of the string-inspired supergravity models. It is also important that the divergent corrections to gaugino masses vanish in the limit of the radius modulus SUSY breaking. For the Higgs mass, we have evaluated one-loop contributions from various types of Yukawa interactions. We have particularly found that in the limit of the radius modulus SUSY breaking, the corrections are insensitive to the ultraviolet cutoff of momentum integrals, while those related to the supergravity models depend linearly or quadratically on the cutoff scale. In addition, the radius $R$ dependence of the Higgs mass has been studied. There we have found that even for a finite number of gauge groups, that is, with finite KK modes included, the consistent behaviors emerge in the certain regions of modulus $F$ terms. 


\section{Finiteness of radiative corrections}

\subsection{Large $N$}

We have observed by examining the cutoff and compactification scale dependences that finite radiative corrections appear in the limit of the radius modulus breaking even for a finite number of gauge groups (i.e. finite KK modes). In continuous five-dimensional theory, radiative corrections to Higgs masses are found to become finite if one adopts SUSY breaking with boundary conditions [17]. In this case, it is important to include an infinitely large number of KK modes in summing up loop corrections while preserving five-dimensional supersymmetry. In other words, if one includes the effects of KK modes which are sufficiently heavier than the momentum cutoff, the locality in the extra dimension is recovered [18]. In our model, that is interpreted as involving a large number of gauge groups or taking a lower four-dimensional cutoff. For example, for $N=200$, loop corrections become cutoff independent in the regime $\Lambda R<15$, while the cutoff insensitivity cannot be seen for $N=10$. However we here want to stress that to have finite corrections is not the main point of this work because we only deal with a four-dimensional theory. (For an approach to cutoff-insensitive Higgs masses in product-group gauge theories, see [19].)

In this subsection, we analytically clarify the high-energy behavior of radiative corrections to Higgs scalar masses, especially focusing on the insensitivity to the momentum cutoff. Passing to Euclidean momenta, consider the following typical form of radiative corrections to Higgs masses from bosonic and fermionic KK modes:

$$
I_{b}=|y|^{2} \sum_{n, \pm} \int \frac{d^{4} p}{(2 \pi)^{4}} \frac{1}{p^{2}+m_{n \pm}^{2}}, \quad I_{f}=-2|y|^{2} \sum_{n} \int \frac{d^{4} p}{(2 \pi)^{4}} \frac{1}{p^{2}+m_{n}^{2}}
$$

where the factor 2 in $I_{f}$ has been introduced so that the boson and fermion degrees of freedom are equal. This type of corrections appears, for example, due to Yukawa couplings between bulk matter multiplets and a boundary Higgs field. In fact, we have already encountered it in Eq. (3.23). (That is a reason we have written the coupling as $y$ in the above equations.) A similar analysis can also be performed for gauge interactions. We treat these momentum integrals with the proper-time regularization and then obtain for $I_{b}$

$$
I_{b}=\frac{|y|^{2}}{16 \pi^{2}} \sum_{n, \pm} \int_{0}^{\infty} d t \frac{1}{t^{2}} e^{-t m_{n \pm}^{2}}
$$

and a similar expression for $I_{f}$. An important point is that the integration and summation can be safely exchanged if one introduces a ultraviolet momentum cutoff $\Lambda$ and truncates the KK-mode sum at a finite level. Note that the model in this paper naturally contains a finite number of KK modes. Thus we find

$$
I_{b}=\frac{|y|^{2}}{16 \pi^{2}} \int_{1 / \Lambda^{2}}^{\infty} d t \frac{1}{t^{2}} \sum_{n, \pm} e^{-t m_{n \pm}^{2}}
$$


To see the cutoff dependence of radiative corrections, we examine the beta functions in the sense of Wilsonian renormalization-group equations:

$$
\begin{gathered}
\frac{\partial I_{b(f)}}{\partial \ln \Lambda^{2}}=\frac{\epsilon_{b(f)}|y|^{2}}{16 \pi^{2}} \Lambda^{2} \\
\epsilon_{b}=\sum_{n, \pm} e^{-m_{n \pm}^{2} / \Lambda^{2}}, \quad \epsilon_{f}=2 \sum_{n} e^{-m_{n}^{2} / \Lambda^{2}} .
\end{gathered}
$$

Since we are now interested in a particular relation between finite radiative corrections and the radius modulus breaking, it is appropriate to take a large $N$ limit (the five-dimensional limit) in analyzing the corrections. In this limit, the results in Section 2 show that bosonic and fermionic KK modes have the following form of tree-level mass eigenvalues

$$
m_{n \pm}^{2}=\left(\frac{n}{R} \pm \frac{F_{T}}{2\langle T\rangle}\right)^{2}+\left|\frac{F_{S}}{2\langle S\rangle}\right|^{2}-\left|\frac{F_{T}}{2\langle T\rangle}\right|^{2}, \quad m_{n}^{2}=\left(\frac{n}{R}\right)^{2}
$$

which is valid for vector and matter multiplets up to the second order of $F$ 's (or exact in the dilaton/radius modulus SUSY-breaking cases). With this mass splitting, we show in Fig. 4 the differences between the beta function coefficients $\epsilon_{b}$ and $\epsilon_{f}$, i.e. the cutoff dependences of the radiative corrections for the three typical cases. It is clearly seen from the figure that the

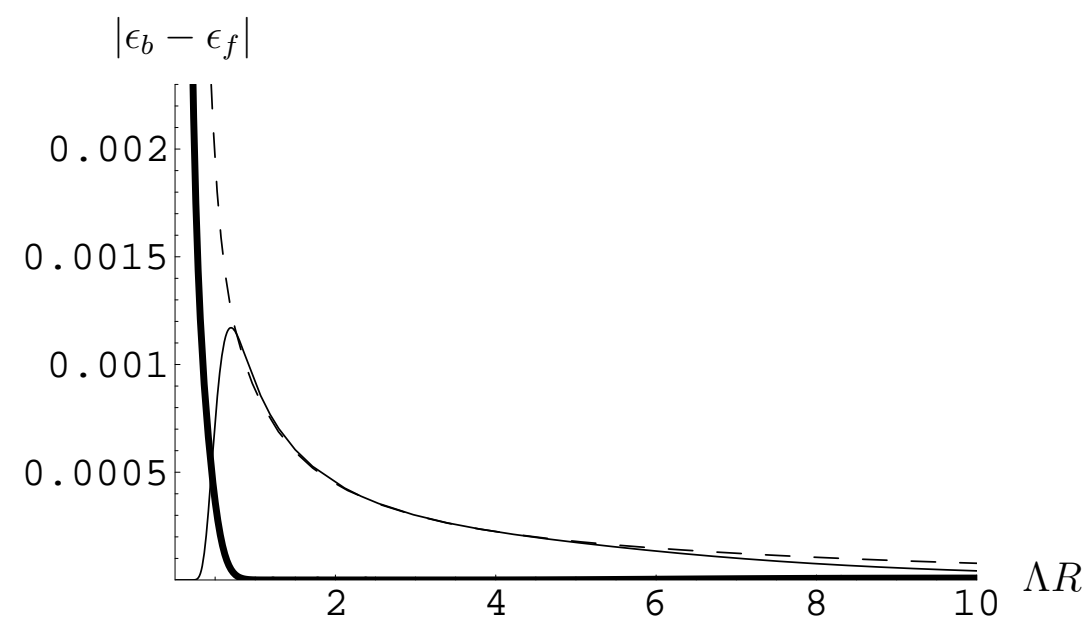

Figure 4: The cutoff dependences of radiative corrections in the large $N$ limit. The horizontal axis denotes the cutoff scale and the vertical one the difference between $\epsilon_{b}$ and $\epsilon_{f}$. We take $F_{S} /\langle S\rangle$ and/or $F_{T} /\langle T\rangle=1 / 10 R$ in the figure. The dashed, solid, and bold lines correspond to the dilaton-, moduli-dominant, and the radius modulus SUSY breaking, respectively.

cutoff dependences are rather different to each other, in particular, the ultraviolet divergence is highly suppressed in the radius modulus breaking case. The dilaton and moduli SUSY breaking have a similar behavior for a large value of the cutoff scale $\Lambda$. This is because 
in these cases the corrections are proportional to the number of KK modes, as mentioned previously.

To analytically study these divergence properties, we estimate the contributions by use of the Poisson formula and obtain

$$
\begin{aligned}
\epsilon_{b} & =2 \sqrt{\pi} \Lambda R \exp \left[\frac{-1}{4 \Lambda^{2}}\left(\left|\frac{F_{S}}{\langle S\rangle}\right|^{2}-\left|\frac{F_{T}}{\langle T\rangle}\right|^{2}\right)\right] \sum_{n} e^{-\frac{\pi^{2} n^{2}}{(\Lambda R)^{2}}} \cos \left(\pi F_{T} R^{2} n\right), \\
\epsilon_{f} & =2 \sqrt{\pi} \Lambda R \sum_{n} e^{-\frac{n^{2}}{(\Lambda R)^{2}}}
\end{aligned}
$$

As can be seen in this expression, for a large value of $\Lambda R$, the zero-mode contributions dominate due to the exponential dumping factors. If assumed that the SUSY-breaking scale is much lower than the compactification radius, the zero-mode contribution approximately gives

$$
\epsilon_{b}^{0}-\epsilon_{f}^{0} \simeq \frac{\sqrt{\pi}}{2} \frac{R}{\Lambda}\left(\left|\frac{F_{T}}{\langle T\rangle}\right|^{2}-\left|\frac{F_{S}}{\langle S\rangle}\right|^{2}\right)
$$

The $\Lambda^{-1}$ dependence of (4.9) implies linearly-divergent corrections to Higgs masses. It can be seen from (4.9) that in the radius modulus breaking case where $F_{S 5} \propto\left(F_{S} /\langle S\rangle+F_{T} /\langle T\rangle\right)=0$, the radiative corrections become cutoff insensitive and therefore finite results follow. On the other hand, the dilaton/moduli dominant cases generally have divergent corrections proportional to $\Lambda R$.

\subsection{Boundary condition breaking}

In continuum five-dimensional theories, it is known that supersymmetry breaking with boundary conditions gives finite radiative corrections to scalar soft masses [17]. This is partly because supersymmetry is broken globally in the bulk and hence the breaking effect is cut off by the size of the extra dimension. On the other hand, in the present four-dimensional model, the finiteness is achieved on a specific line of the SUSY-breaking parameter space. We will show how these two cases are related. To this end, we formulate the 'boundary' condition breaking of supersymmetry in out setup and examine whether the resulting spectrum matches to that of the radius modulus breaking $\left(F_{S 5}=0\right)$. If these two spectra are found to be equivalent, they may be transformed to each other by unitary transformation, which gives a support that radiative corrections are ultraviolet finite.

\subsubsection{Without twists}

Before discussing the boundary condition breaking, it is useful to remind how to compactify the latticized extra dimensions. To begin with, suppose that there are an infinite number of gauge groups and corresponding matter fields. Compactifying physical space is performed with the identification of indices under $i \rightarrow i+N$, which is interpreted as a coordinate translation in the extra dimension. On the other hand, the identification on the field space, i.e. boundary conditions under this translation is given by $\Phi_{i+N} \equiv \Phi_{i}$. These procedures result in leaving the 
$N$ copies of gauge groups and matter fields as physical degrees of freedom. The identification on the field space is clearly seen by recombining the fields as

$$
\Phi_{j}=\frac{1}{\sqrt{N}} \sum_{k=0}^{N-1}\left(\omega_{k}\right)^{j} \widetilde{\Phi}_{k},
$$

where $\omega_{k} \equiv e^{\frac{2 \pi k}{N} i}$, which is just the Fourier mode expansion in the continuum limit. Note that there are only finite numbers of mass eigenstates $\widetilde{\Phi}_{k}$, because the model comes back to higher dimensions only in the large $N$ limit. At this stage, it is straightforward to include various types of twisted boundary conditions. In what follows, we will study a specific case which is related to the radius modulus breaking of supersymmetry.

\subsection{2 $S U(2)$ twist}

Along the line discussed above, we demonstrate the twisted boundary condition for vector multiplets as an illustrative example. The field content we consider is given by Table (2.1) except that we now have an infinite numbers of gauge groups and $Q$ fields. In particular, there are two types of spinors $\lambda$ and $\chi$ for every gauge group. The identification of physical space is the same as the above untwisted case. A new ingredient now introduced is the boundary condition on the field space. Let us consider the following $S U(2)$ twist upon the identification of field space;

$$
\left(\begin{array}{c}
\lambda_{j+N} \\
\chi_{j+N}
\end{array}\right)=e^{2 \pi i \theta_{a} \sigma_{a}}\left(\begin{array}{c}
\lambda_{j} \\
\chi_{j}
\end{array}\right)
$$

where $\sigma_{a}$ are the Pauli's matrices. The parameters $\theta_{a}$ will turn out to be SUSY-breaking order parameters. The mass matrix of these spinor fields must be consistent with this identification and therefore takes the form (for small $\theta_{a}$ )

$$
\begin{aligned}
& v(\lambda \mid \chi)\left(M_{0}+\delta M\right)\left(\frac{\lambda}{\chi}\right)+\text { h.c. },
\end{aligned}
$$

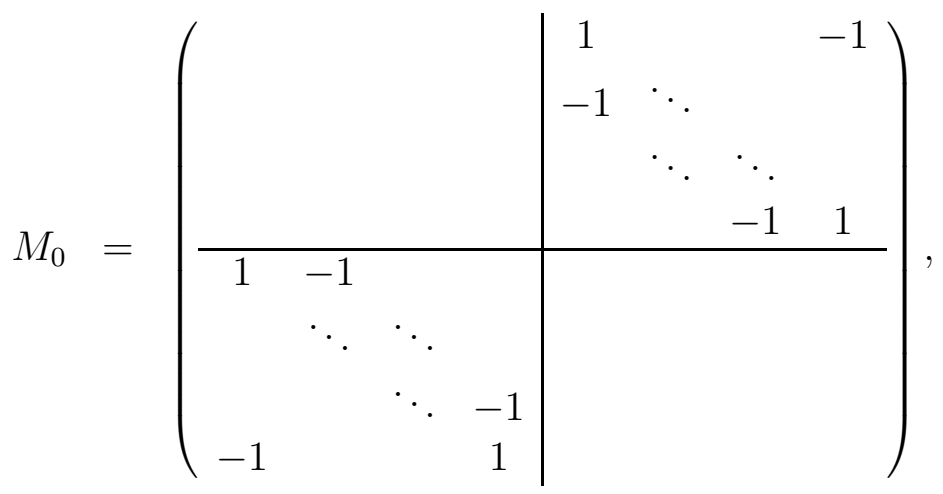




$$
\delta M=\left(\begin{array}{l|lc} 
& & -2 \pi i \theta_{3} \\
& & \\
-2 \pi i \theta_{3} & -2 \pi\left(i \theta_{1}+\theta_{2}\right) &
\end{array}\right) .
$$

The $M_{0}$ part denotes the mass matrix for untwisted fields, whose eigenvalues are given by (2.4). On the other hand, $\delta M$ arises due to the twisting boundary condition (4.11). These mass terms become the kinetic energy along the extra dimension if one takes the continuum limit.

Let us diagonalize the mass matrix by perturbation theory with respect to $\theta_{a}$. The eigenvalues and eigenmodes of the unperturbed matrix $M_{0}$ are given by

$$
m_{k \pm}^{(0)}= \pm 2 v \sin \left(\frac{k \pi}{N}\right), \quad \widetilde{\psi}_{k}^{ \pm}=\frac{1}{\sqrt{2 N}} \sum_{j=1}^{N}\left(\omega_{k}\right)^{j}\left( \pm \lambda_{j}+\chi_{j}\right) .
$$

The irrelevant overall phases factors have been absorbed into field redefinition. In perturbation theory, the nonzero matrix elements of $\delta M$ linear in $\theta$ 's are

$$
\left\langle\widetilde{\psi}_{k}^{+}|\delta M| \widetilde{\psi}_{k}^{+}\right\rangle=\left\langle\widetilde{\psi}_{k}^{-}|\delta M| \widetilde{\psi}_{k}^{-}\right\rangle=\frac{-2 \pi}{N} \cos \left(\frac{2 \pi k}{N}\right)\left(i \theta_{1}+\theta_{2}+i \theta_{3}\right),
$$

and then the eigenvalues at the first order become

$$
m_{k \pm}^{(1)}=m_{k \pm}^{(0)}-\frac{2 \pi v}{N} \cos \left(\frac{2 \pi k}{N}\right)\left(i \theta_{1}+\theta_{2} \pm i \theta_{3}\right) .
$$

The first term on the right-handed side means the KK masses and therefore the second one is interpreted as SUSY-breaking effect. For example, in the case that $\theta_{1}, \theta_{2} \neq 0$ and $\theta_{3}=0$, the mass eigenvalues for the low-lying modes $(k \ll N)$ read

$$
m_{k \pm}^{(1)} \simeq \pm \frac{k}{R}-\frac{F_{T}}{2 R}
$$

where the SUSY-breaking order parameter is identified as $F_{T} \equiv 2\left(i \theta_{1}+\theta_{2}\right)$. This is indeed the spectrum that is given by the radius modulus breaking (2.15). Therefore the finiteness property of radiative corrections in this case is now understood.

For $\theta_{3}=0$, a half of supersymmetry in five dimensions is left unbroken, namely, a linear combination of the zero-mode gauge fermions is massless, which composes a four-dimensional massless vector multiplet together with the gauge boson zero mode. When $\theta_{3}$ is turned on as well as $\theta_{1,2}$, the remaining $N=1$ supersymmetry is broken. This corresponds to applying the twisted boundary condition with a $U(1)$ rotation which does not commute with the $N=1$ supersymmetry. 
It may be interesting to rewrite the mass term (4.12) in terms of the fields with untwisted boundary conditions. We consider for simplicity the twisting with $\sigma_{2}$. It is straightforward to generalize the following result to the cases with generic types of twisting. Let us redefine the spinor fields as

$$
\left(\begin{array}{l}
\lambda_{j} \\
\chi_{j}
\end{array}\right)=e^{2 \pi i \theta_{2} \frac{j}{N} \sigma_{2}}\left(\begin{array}{l}
\lambda_{j}^{\prime} \\
\chi_{j}^{\prime}
\end{array}\right) .
$$

It can be easily checked that the fields with primes satisfy the untwisted boundary condition under the translation $j \rightarrow j+N$,

$$
\left(\begin{array}{l}
\lambda_{j+N}^{\prime} \\
\chi_{j+N}^{\prime}
\end{array}\right)=\left(\begin{array}{l}
\lambda_{j}^{\prime} \\
\chi_{j}^{\prime}
\end{array}\right) .
$$

Almost all terms in the Lagrangian are invariant under this field redefinition, while the mass term (4.12) is not. This means the kinetic term along the fifth dimension is not invariant under the coordinate-dependent phase rotation. In the basis of $\lambda^{\prime}$ and $\chi^{\prime}$, the mass matrix becomes for small $\theta$,

$$
\begin{aligned}
& (4.12) \simeq\left(\lambda^{\prime} \mid \chi^{\prime}\right) M_{0}\left(\frac{\lambda^{\prime}}{\chi^{\prime}}\right)-\frac{2 \pi \theta_{2} v}{N}\left(\begin{array}{lll}
\lambda_{1}^{\prime} & \cdots & \lambda_{N}^{\prime}
\end{array}\right)\left(\begin{array}{cccc} 
& 1 & & \\
& & \ddots & \\
& & & 1 \\
& & &
\end{array}\right)\left(\begin{array}{c}
\lambda_{1}^{\prime} \\
\vdots \\
\vdots \\
\lambda_{N}^{\prime}
\end{array}\right)
\end{aligned}
$$

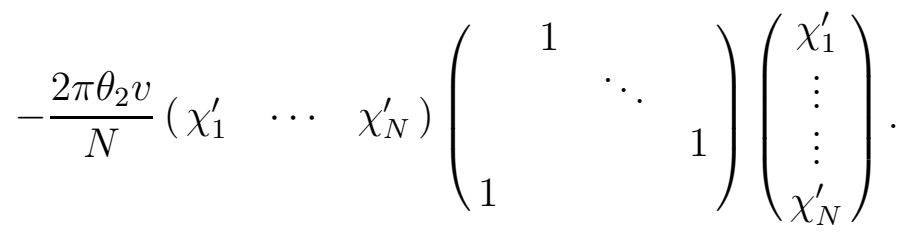

Diagonalizing this matrix should lead to the eigenvalues (4.18) with $\theta_{1}=0$. The first term in the right-handed side is the kinetic energy term along the fifth dimension. The second and third terms can be interpreted as the mass terms which come from a VEV of extra component of gauge field [20]. That is, these three terms together make up the covariant derivative of the spinor fields. It was shown [16] that the radius modulus breaking of supersymmetry is equivalent to the Wilson line breaking, proving that a VEV of the extra component of graviphoton field generates SUSY breaking in the five-dimensional off-shell supergravity (as seen in the second and third terms in the above). The present analysis makes it clear from a four-dimensional viewpoint that the relevant $\mathrm{VEV}$ is that of the modulus $Q\left(F_{Q} \propto F_{T}\right)$.

\section{Summary}

In this paper, we have formulated four-dimensional SUSY breaking in product-group gauge theories. The model contains several modulus fields corresponding to the dilaton and the radion. The modulus fields satisfy the specific relations suggested by some correspondences to higher-dimensional physics. From these, the relations are extracted for the $F$-component 
VEVs of modulus fields. The non-trivial moduli dependences of the action have also been determined. We have shown that at intermediate energy regime, the mass spectra of typical SUSY breaking scenarios (the dilaton/moduli dominance and the radius modulus breaking) appear in the corresponding limits on the space of SUSY-breaking order parameters. We have calculated in detail the gaugino and Higgs scalar masses up to one-loop level. Our results seem to be consistent with various aspects of bulk SUSY breaking, e.g. the string-inspired supergravity models.

The cutoff dependences of loop corrections have been investigated in detail. We have calculated the gaugino and Higgs mass corrections from various types of gauge and Yukawa couplings. In particular, we have shown that the insensitivity to ultraviolet cutoff emerges for the radius modulus breaking case. However, for other cases, the spectrum depends linearly or quadratically on the momentum cutoff. This can be understood as the number of KK modes running in the loop diagrams. The compactification radius dependence of the one-loop mass spectrum has also been studied.

The finiteness property of radiative corrections has been examined from several different viewpoints. In particular, we have formulated in our setup the boundary condition breaking of supersymmetry (or the Hosotani mechanism) and shown that the spectrum indeed agrees with that of the radius modulus breaking case. This result indicates that the obtained finite corrections are due to the global breaking of symmetries in the bulk.

While, in this work, we have focused on the special limits corresponding to higher-dimensional SUSY breaking, it will be interesting to investigate other regions of modulus $F$ terms. Such a generic pattern of $F$ terms might induce sparticle mass spectra not yet explored in the literature. It is also possible to extend the present analysis to include brane matter which is interpreted as being charged under one of the gauge groups $G_{i}$ 's. The SUSY-breaking masses of this type of fields depend on whether they can couple to the moduli $S$ and $Q$. However there seems to be, in general, few principles to fix modulus couplings of brane fields, and the couplings would also depend on more fundamental theories. The presence of brane fields may be useful to generate Yukawa hierarchy of quarks and leptons. Explicit four-dimensional model construction along this line leads concrete predictions of (super) particle spectrum, compared to those of other models. The model parameters might then be constrained by clarifying spectrum and applying it to supersymmetric standard models, etc. We leave such phenomenological analyses to future work.

\section{Acknowledgments}

The authors would like to thank Tatsuo Kobayashi for a collaboration at an early stage of this work and lots of valuable discussions. This work is supported in part by the Special Postdoctoral Researchers Program at RIKEN. 


\section{References}

[1] I. Antoniadis, Phys. Lett. 246B (1990) 377; N. Arkani-Hamed, S. Dimopoulos and G. Dvali, Phys. Lett. 429B (1998) 263; I. Antoniadis, N. Arkani-Hamed, S. Dimopoulos and G. Dvali, Phys. Lett. 436B (1998) 257.

[2] L. Randall and R. Sundrum, Phys. Rev. Lett. 83 (1990) 3370; ibid. 83 (1999) 4690.

[3] N. Arkani-Hamed, A.G. Cohen and H. Georgi, Phys. Rev. Lett. 86 (2001) 4757.

[4] C.T. Hill, S. Pokorski and J. Wang, Phys. Rev. D64 (2001) 105005.

[5] H.C. Cheng, C.T. Hill, S. Pokorski and J. Wang, Phys. Rev. D64 (2001) 065007; A. Sugamoto, Prog. Theor. Phys. 107 (2002) 793; N. Arkani-Hamed, A.G. Cohen and H. Georgi, Phys. Lett. 513B (2001) 232; H.C. Cheng, C.T. Hill and J. Wang, Phys. Rev. D64 (2001) 095003; M. Bander, Phys. Rev. D64 (2001) 105021; C. Csaki, G.D. Kribs and J. Terning, Phys. Rev. D65 (2002) 015004; H.C. Cheng, K.T. Matchev and J. Wang, Phys. Lett. 521B (2001) 308; C.T. Hill, Phys. Rev. Lett. 88 (2002) 041601; N. Arkani-Hamed, A.G. Cohen and H. Georgi, JHEP 0207 (2002) 020; I. Rothstein and W. Skiba, Phys. Rev. D65 (2002) 065002; P.H. Chankowski, A. Falkowski and S. Pokorski, JHEP 0208 (2002) 003; N. Arkani-Hamed, A.G. Cohen, D.B. Kaplan, A. Karch and L. Motl, JHEP 0301 (2003) 083; W. Skiba and D. Smith, Phys. Rev. D65 (2002) 095002; R. S. Chivukula and H. J. He, Phys. Lett. 532B (2002) 121; Z. Berezhiani, A. Gorsky and I.I. Kogan, JETP Lett. 75 (2002) 530; T.j. Li and T. Liu, Eur.Phys.J.C 28 (2003) 545; C. T. Hill and A. K. Leibovich, Phys. Rev. D66 (2002) 016006; N. Arkani-Hamed, A. Cohen, E. Katz, A.E. Nelson, T. Gregoire and J. Wacker, JHEP 0208 (2002) 021; C. Csaki, J. Erlich and J. Terning, Phys. Rev. D67 (2003) 025019; S. Chang and H. Georgi, Nucl. Phys. B672 (2003) 101; N. Arkani-Hamed, H. Georgi and M.D. Schwartz, Annals Phys. 305 (2003) 96; A. Falkowski, H.P. Nilles, M. Olechowski and S. Pokorski, Phys. Lett. 566B (2003) 248; J. Giedt, E. Poppitz and M. Rozali, JHEP 0303 (2003) 035; N. Arkani-Hamed and M.D. Schwartz, hep-th/0302110; P. Brax, R.A. Janik and R. Peschanski, Nucl. Phys. B660 (2003) 194; E. Dudas, A. Falkowski and S. Pokorski, Phys. Lett. 568B (2003) 281; K.R.S. Balaji, M. Lindner and G. Seidl, Phys. Rev. Lett. 91 (2003) 161803; D.T. Son and M.A. Stephanov, hep-ph/0304182; E. Poppitz, JHEP 0308 (2003) 044; A. Falkowski, C. Grojean and S. Pokorski, hep-ph/0310201.

[6] T. Kobayashi, N. Maru and K. Yoshioka, Eur. Phys. J. C29 (2003) 277.

[7] K. Sfetsos, Nucl. Phys. B612 (2001) 191; H. Abe, T. Kobayashi, N. Maru and K. Yoshioka, Phys. Rev. D67 (2003) 045019; A. Falkowski and H.D. Kim, JHEP 0208, 052 (2002); L. Randall, Y. Shadmi and N. Weiner, JHEP 0301 (2003) 055.

[8] C. Csaki, J. Erlich, V. V. Khoze, E. Poppitz, Y. Shadmi and Y. Shirman, Phys. Rev. D65 (2002) 085033. 
[9] C. Csaki, J. Erlich, C. Grojean and G. Kribs, Phys. Rev. D65 (2002) 015003; H. C. Cheng, D. E. Kaplan, M. Schmaltz and W. Skiba, Phys. Lett. 515B (2001) 395.

[10] L.E.Ibáñez and D. Lüst, Nucl. Phys. B382 (1992) 305; B. de Carlos, J.A. Casas and C. Muñoz, Phys. Lett. 299B (1993) 234; A. Brignole, L.E.Ibáñez and C. Muñoz, Nucl. Phys. B422 (1994) 125; T. Kobayashi, D. Suematsu, K. Yamada and Y. Yamagishi, Phys. Lett. 348B (1995) 402.

[11] E. Cremmer, S. Ferrara, C. Kounnas and D.V. Nanopoulos, Phys. Lett. 133B (1983) 61; J.R. Ellis, A.B. Lahanas, D.V. Nanopoulos, and K. Tamvakis, Phys. Lett. 134B (1984) 429; J.R. Ellis, C. Kounnas, D.V. Nanopoulos, Nucl. Phys. B247 (1984) 373; A.B. Lahanas and D.V. Nanopoulos, Phys. Rept. 145 (1987) 1.

[12] Z. Chacko and M.A. Luty, JHEP 0105 (2001) 067; D. Marti and A. Pomarol, Phys. Rev. D64 (2001) 105025.

[13] T. Kobayashi and K. Yoshioka, Phys. Rev. Lett. 85 (2000) 5527.

[14] G.F. Giudice and R. Rattazzi, Nucl. Phys. B511 (1998) 25.

[15] J. Scherk and J.H. Schwarz, Phys. Lett. 82B, 60 (1979); Nucl. Phys. B153 (1979) 61.

[16] G. von Gersdorff and M. Quiros, Phys. Rev. D65 (2002) 064016.

[17] I. Antoniadis, S. Dimopoulos, A. Pomarol and M. Quiros, Nucl. Phys. B544 (1999) 503; R. Barbieli, L. Hall and Y. Nomura, Phys. Rev. D63 (2001) 105007; N. Arkani-Hamed, L.J. Hall, Y. Nomura and D.R. Smith, Nucl. Phys. B605 (2001) 81.

[18] T. Kobayashi and H. Terao, Prog. Theor. Phys. 107 (2002) 785.

[19] A. Falkowski, C. Grojean and S. Pokorski, Phys. Lett. 535B (2002) 258.

[20] Y. Hosotani, Phys. Lett. 126B (1983) 309; Ann. Phys. 190 (1989) 233. 